\title{
Large and infinite extra dimensions
}

\author{
V. A. Rubakov 网 \\ Institute for Nuclear Research of the Russian Academy of Sciences, 60th October \\ Anniversary Prospect, 7a, Moscow, 117312, Russia
}

\begin{abstract}
The emphasis in the developmet of theories with more than three spatial dimensions has recently shifted towards "brane world" picture, which assumes that ordinary matter (with possible exceptions of gravitons and other, hypothetic, particles which interact very weakly with matter) is trapped to a three-dimensional submanifold brane - embedded in fundamental multi-dimensional space. In the brane world scenario, extra dimensions may be large, and even infinite; they may have effects, directly observable in current or fothcoming experiments. On the basis of simple field-theoretic models, various ideas in this direction are exposed at a non-expert level.
\end{abstract}

\section{Contents}

\begin{tabular}{|cc}
\hline Introduction & 1
\end{tabular}

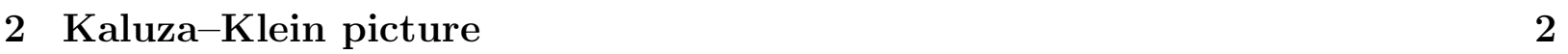

$\begin{array}{lll}3 & \text { Localized matter } & 3\end{array}$

3.1 Localized fermions . . . . . . . . . . . . . . . . . . . . . . . . . . . . . . 3

3.2 Localized gauge fields . . . . . . . . . . . . . . . . . . . . . . . 6

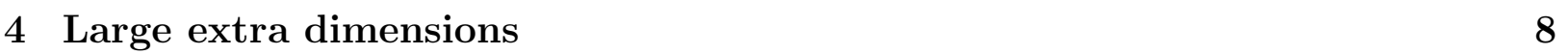

$4.1 \quad$ Size of extra dimensions $\ldots \ldots \ldots$. . . . . . . . . . . . . . . . . 8

$4.2 \quad$ Light KK gravitons: colliders, cosmology and astrophysics . . . . . . . . . . 10

4.3 Sterile neutrinos in the bull . . . . . . . . . . . . . . . . . . . . . 15

4.4 Unification of couplings . . . . . . . . . . . . . . . . . . . . . 16

4.5 Problem with proton stability . . . . . . . . . . . . . . . . . . . . 20

5 Non-factorizable geometry 20

5.1 Warped extra dimension . . . . . . . . . . . . . . . . . 21

5.2 Two-brane set up . . . . . . . . . . . . . . . . . . . . . . . . 23

5.3 Matter on negative tension brane and hierarchy . . . . . . . . . . . . . . . 25

5.4 Matter on positive tension brand $\ldots \ldots \ldots \ldots$. . . . . . . . . . . 27

*E-mail: rubakov@ms2.inr.ac.ru 
$\begin{array}{lll}6 & \text { Infinite extra dimension } & 27\end{array}$

6.1 Localized graviton . . . . . . . . . . . . . . . . . . . . . . . . 27

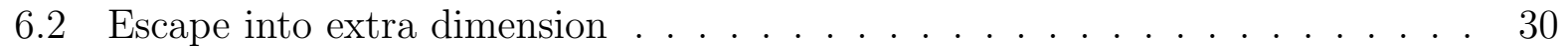

6.3 Holographic interpretation . . . . . . . . . . . . . . . . . . . . . 34

6.4 More than one extra dimensions. Localization of gauge fields by gravity . . . 35

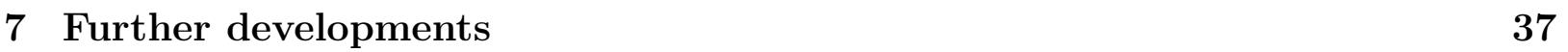

7.1 Cosmological constant . . . . . . . . . . . . . . . . . . . . . . . . . . . . . 38

7.2 Gravity at ultra-large distances . . . . . . . . . . . . . . . . . . . . . . . . . 39

7.3 Approximate Lorentz invariance . . . . . . . . . . . . . . . . . . . . . . . . . 41

7.4 Creation of a brane Universe . . . . . . . . . . . . . . . . . . . . . 43

8 Conclusion $\quad 45$ 


\section{Introduction}

The possibility that our space has more than three spatial dimensions has been attracting continuing interest for many years. Strong motivation for considering space as multidimensional comes from theories which incorporate gravity in a reliable manner — string theory and M-theory: almost all their versions are naturally and/or consistently formulated in space-time of more than four dimensions. In parallel to developments in the fundamental theory, studies along more phenomenological lines have recently lead to new insights on whether and how extra dimensions may manifest themselves, and whether and how they may help to solve long-standing problems of particle theory (hierarchy problem, cosmological constant problem, etc.). These phenomenological studies are often based on simplified (even over-simplified) field-theoretic models, and this approach has its advantages and disadvantages. An advantage is that, by considering various models, one reveals a whole spectrum of possibilities. A disadvantage is that some (most?) of these models may have nothing to do with fundamental theory, so it is unclear which of these possibilities have a chance to be realized in nature. Furthermore, quantitative estimates are often at best order-ofmagnitude, and in many cases are not available at all, as most of these models have free parameters. Still, the phenomenological approach, which is the subject of this mini-review, helps to understand how to search for extra dimensions, if there exist ones.

An important issue in multi-dimensional theories is the mechnaism by which extra dimensions are hidden, so that the space-time is effectively four-dimensional insofar as known physics is concerned. Until recently, the main emphasis was put on theories of KaluzaKlein type, where extra dimensions are compact and essentially homogeneous. In this picture, it is the compactness of extra dimensions that ensures that space-time is effectively four-dimensional at distances exceeding the compactification scale (size of extradimensions). Hence, the size of extra dimensions must be microscopic; a "common wisdom" was that this size was roughly of the order of the Planck scale (although compactifications at the electroweak scale were also considered, see. e.g., [1, 2, 3]). With the Planck length $l_{P l} \sim 10^{-33} \mathrm{~cm}$ and the corresponding energy scale $M_{P l} \sim 10^{19} \mathrm{GeV}$, probing extra dimensions directly appeared hopeless.

Recently, however, the emphasis has shifted towards "brane world" picture which assumes that ordinary matter (with possible exceptions of gravitons and other, hypothetic, particles which interact very weakly with matter) is trapped to a three-dimensional submanifold - brane - embedded in fundamental multi-dimensional space. In the brane world scenario, extra dimensions may be large, and even infinite; we shall see that they may then have experimentally observable effects.

Certainly, the potential detectability of large and infinite extra dimensions is one of the reasons of why they are interesting. Another reason is that lower-dimensional manifolds, $p$-branes, are inherent in string/M-theory. Some kinds of $p$-branes are capable of carrying matter fields; for example, $D$-branes have gauge fields residing on them (for a review, see Ref. [4). Hence, the general idea of brane world appears naturally in M-theory context, and, indeed, realistic brane-world models based on M-theory have been proposed [5, 6]. Even 
though the phenomenological models to be discussed in this mini-review may have nothing to do with M-theory $p$-branes, one hopes that some of their properties will have counterparts in the fundamental theory. We note in this regard that the term "brane" has quite different meaning in different contexts; we shall use this term for any three-dimensional submanifold to which ordinary matter is trapped, irrespectively of the trapping mechanism.

The purpose of this mini-review is to expose, on the basis of simple models, some ideas and results related to large and infinite extra dimensions. We do not attempt at a comprehensive discussion of such constructions; our choice of topics will thus be very personal, and the list of references very incomplete. Neither are we going to present historical overview; a view on history of the brane world scenario is presented, e.g., in Ref. [7].

\section{Kaluza-Klein picture}

To begin with, let us outline the basic idea of the Kaluza-Klein scenario; this will serve as a point of reference for further discussions. The simplest case is one extra spatial dimension $z$, so that the complete set of coordinates in $(4+1)$-dimensional space-time is $\left(x^{\mu}, z\right), \mu=$ $0,1,2,3$. The low energy physics will be effectively four-dimensional if the coordinate $z$ is compact with a certain compactification radius $R$. This means that $z$ runs from 0 to $2 \pi R$, and points $z=0$ and $z=2 \pi R$ are identified. In other words, the four-dimensional space is a cylider whose three dimensions $x^{1}, x^{2}, x^{3}$ are infinite, and the fourth dimension $z$ is a circle of radius $R$. Assuming that this cylinder is homogeneous, and that the metric is flat, one writes a complete set of wave functions of a free massless particle on this cylinder (e.g., solutions to five-dimensional Klein-Gordon equation),

$$
\phi_{\mathbf{p}, n}=\mathrm{e}^{i p_{\mu} x^{\mu}} \mathrm{e}^{i n z / R}, \quad n=0, \pm 1, \pm 2, \ldots .
$$

Here $p_{\mu}$ is the $(3+1)$-dimensional momentum and $n$ is the eigenvalue of (one-dimensional) angular momentum. Since $\phi(x, z)$ obeys $\square_{(5)} \phi=0$, these quantities are related,

$$
p_{\mu} p^{\mu}-\frac{n^{2}}{R^{2}}=0
$$

Hence, inhomogeneous modes with $n \neq 0$ carry energy of order $1 / R$, and they cannot be excited in low energy processes. Below the energy scale $1 / R$, only homogeneous modes with $n=0$ are relevant, and low energy physics is effectively four-dimensional.

From (3+1)-dimensional point of view, each Kaluza-Klein (KK) mode can be interpreted as a separate type of particle with mass $m_{n}=|n| / R$, according to eq. (1). Every multi-dimensional field corresponds to a Kaluza-Klein tower of four-dimensional particles with increasing masses. At low energies, only massless (on the scale $1 / R$ ) particles can be produced, whereas at $E \sim 1 / R$ extra dimensions will show up. Since the KK partners of ordinary particles (electrons, photons, etc.) have not been observed, the energy scale $1 / R$ must be at least in a few hundred GeV range, so in the Kaluza-Klein scenario, the size of extra dimensions must be microscopic $\left(R \lesssim 10^{-17} \mathrm{~cm}\right)$. These properties are inherent in all 
models of Kaluza-Klein type (with larger number of extra dimensions, compactifications on non-trivial manifolds or orbifolds instead of a circle, etc.).

\section{Localized matter}

To see that it is indeed conceivable that ordinary matter may be trapped to a brane, we present in this section simple field-theoretic models exhibiting this property. Throughout this section we neglect gravity; new possibilities emerging when gravitational interactions are included will be considered later on.

\subsection{Localized fermions}

It is fairly straightforward to construct field-theoretic models with localized fermions. The simplest model of this sort has one extra dimension $z$, with the brane being a domain wall [8] (see also Ref. [9]). Namely, let us consider a theory of one real scalar field $\varphi$ whose action is

$$
S_{\varphi}=\int d^{4} x d z\left[\frac{1}{2}\left(\partial_{A} \varphi\right)^{2}-V(\varphi)\right]
$$

Here the subscript $A$ denotes all five coordinates, and the scalar potential $V(\varphi)$ has a double-well shape with two degenerate minima at $\varphi= \pm v$, as shown in Fig. 1.

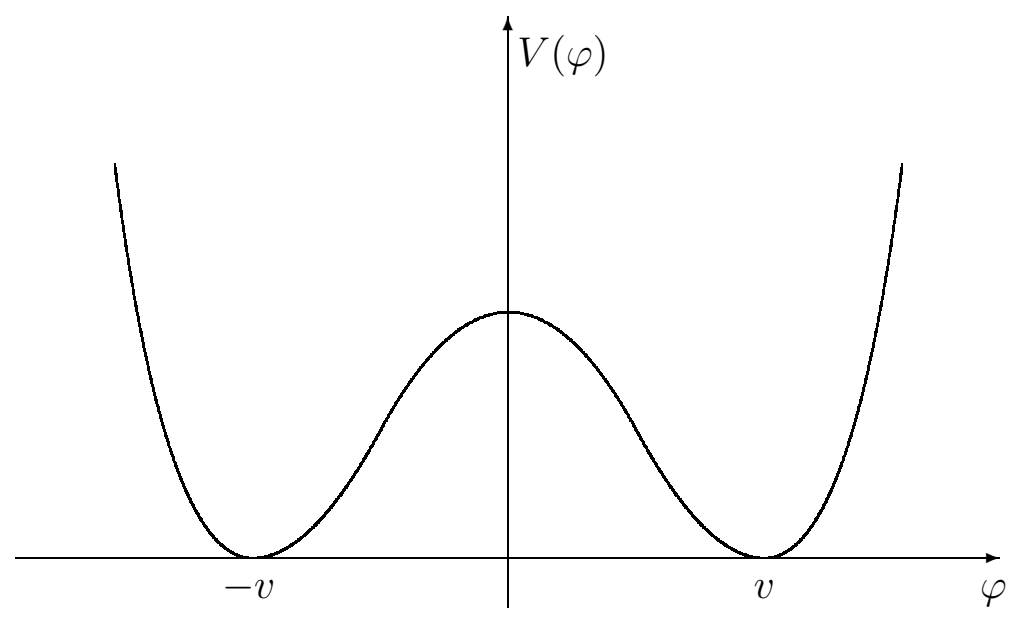

Figure 1: Scalar potential in the model (2).

There exists a classical solution $\varphi_{c}(z)$, kink, depending on one coordinate only. This solution is sketched in Fig. 2. It has asymptotics

$$
\varphi_{c}(z \rightarrow+\infty)=+v
$$




$$
\varphi_{c}(z \rightarrow-\infty)=-v
$$

and describes a domain wall separating two classical vacua of the model. Obviously, the field $\varphi_{c}(z)$ breaks translational invariance along the extra dimension, but leaves the fourdimensional Poincaré invariance intact.

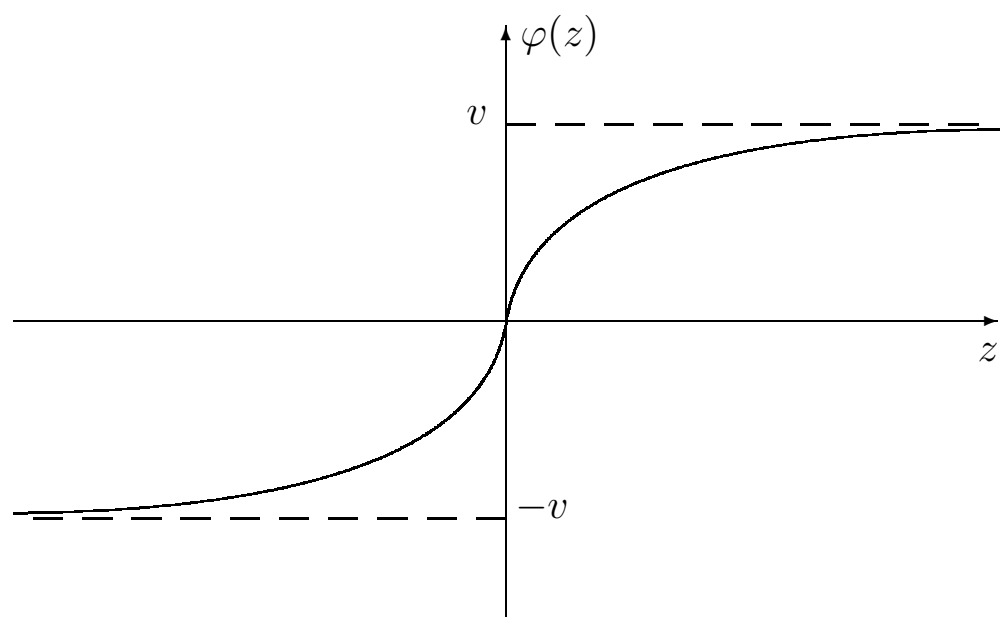

Figure 2: Domain wall solution.

Let us now introduce fermions into this model. We recall that fermions in five-dimensional space-time are four-component columns, and that the five-dimensional gamma-matrices can be chosen as follows,

$$
\begin{gathered}
\Gamma^{\mu}=\gamma^{\mu}, \quad \mu=0,1,2,3, \\
\Gamma^{z}=-i \gamma^{5}
\end{gathered}
$$

where $\gamma^{\mu}$ and $\gamma^{5}$ are the standard Dirac matrices of four-dimensional theory. Introducing the Yukawa interaction of fermions with the scalar field $\varphi$, we write the five-dimensional action for fermions,

$$
S_{\Psi}=\int d^{4} x d z\left(i \bar{\Psi} \Gamma^{A} \partial_{A} \Psi-h \varphi \bar{\Psi} \Psi\right)
$$

Note that in each of the scalar field vacua, $\varphi= \pm v$, five-dimensional fermions acquire a mass

$$
m_{5}=h v
$$

Let us consider fermions in the domain wall background. The corresponding Dirac equation is

$$
i \Gamma^{A} \partial_{A} \Psi-h \varphi_{c}(z) \Psi=0
$$

Due to unbroken four-dimensional Poincaré invariance, the fermion wave functions may be characterized by four-momentum $p_{\mu}$, and we are interested in the spectrum of fourdimensional masses $m^{2}=p_{\mu} p^{\mu}$. A key point is that there exists a zero mode [10, a solution 
to eq. (4) with $m=0$. For this mode one has $\gamma^{\mu} p_{\mu} \Psi_{0}=0$, and the Dirac equation (4) becomes

$$
\gamma^{5} \partial_{z} \Psi_{0}=h \varphi_{c}(z) \Psi_{0}
$$

The zero mode is left-handed from the four-dimensional point of view,

$$
\gamma_{5} \Psi_{0}=-\Psi_{0}
$$

and has the form

$$
\Psi_{0}=\mathrm{e}^{-\int_{0}^{z} d z^{\prime} h \varphi_{c}\left(z^{\prime}\right)} \psi_{L}(p)
$$

where $\psi_{L}(p)$ is the usual solution of the four-dimensional Weyl equation. The zero mode (5) is localized near $z=0$, i.e., at the domain wall, and at large $|z|$ it decays exponentially, $\Psi_{0} \propto \exp \left(-m_{5}|z|\right)$.

The spectrum of four-dimensional masses is shown in Fig. 3. Besides the chiral zero mode, there may or may not exist bound states, but in any case the masses of the latter are proportional to $v$ and are large for large $v$. There is also a continuum part of the spectrum starting at $m=m_{5}$; the continuum states correspond to five-dimensional fermions which are not bound to the domain wall and escape to $|z|=\infty$.

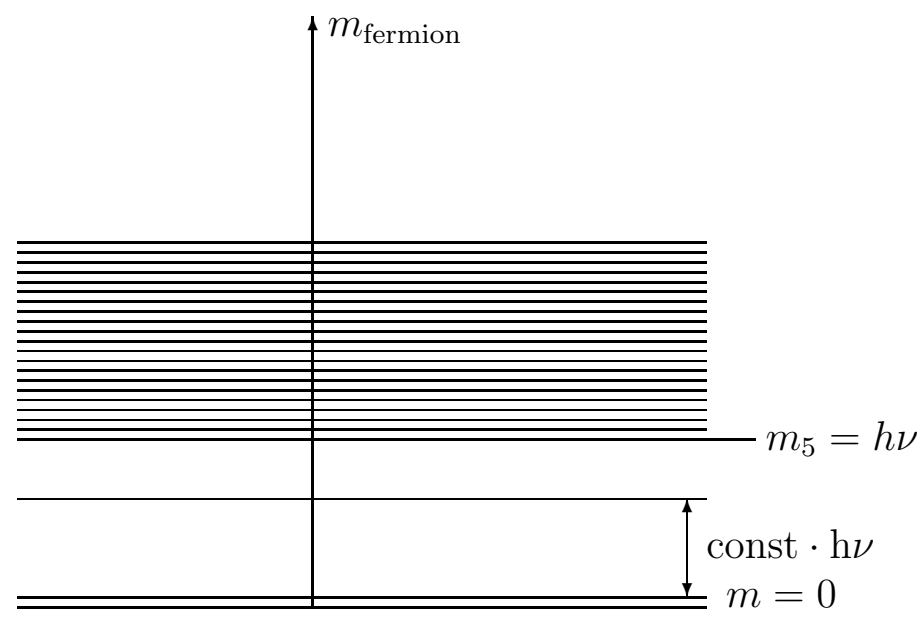

Figure 3: Spectrum of four-dimensional masses of fermions in domain wall background. The gap between zero mode (with $m=0$ ) and non-zero modes is of order $h v$. Continuum starts at $m=m_{5} \equiv h v$.

Massless four-dimensional fermions localized on the domain wall, zero modes, are meant to mimick our matter. They propagate with the speed of light along the domain wall, but do not move along $z$. Of course, in realistic theories they should acquire small masses by one or another mechanism. At low energies, their interactions can produce only zero modes again, so physics is effectively four-dimensional. Zero modes interacting at high 
energies, however, will produce continuum modes, the extra dimension will open up, and particles will be able to leave the brane, escape to $|z|=\infty$ (if the size of extra dimension is infinite) and literally disappear from our world. For a four-dimensional observer (composed of particles trapped to the brane), these high energy processes will look like $e^{+} e^{-} \rightarrow$ nothing or $e^{+} e^{-} \rightarrow \gamma+$ nothing. We shall discuss later on whether these and similar processes are indeed possible when gravitational and gauge interactions are taken into account, whether they may lead to apparent non-conservation of energy, electric charge, etc.

The above construction is straightforwardly generalized to more than one extra dimensions. This is done by considering, instead of the domain wall, topological defects of higher codimensions: the Abrikosov-Nielsen-Olesen vortex in six-dimensional space-time $(D=6$, number of extra dimensions $d=2$ ), 't Hooft-Polyakov monopole in $D=7(d=3)$, etc. In many cases, the existence of fermion zero modes in the background of the topological defect is guaranteed by the corresponding index theorem. Explicit expressions for fermion zero modes in various backgrounds are given in Refs. [10, 11, 12. As a bonus, the fourdimensional massless fermions localized on topological defects are usually chiral. Furthermore, the number of fermion zero modes may be greater than one, so from one family of multi-dimensional fermions one can obtain several four-dimensional families. This possibility of explaining the origin of three Standard Model generations has been explored in Refs. [13, 14 where it has been found that reasonable pattern of masses and mixings can be obtained in a fairly natural way.

\subsection{Localized gauge fields}

Localizing gauge fields on a brane is more difficult. The mechanism just described does not have chance to work, at least for massless non-Abelian fields. The reason is as follows. If the gauge field had a zero mode whose wave function $A(z)$ is localized near the brane, the four-dimensional effective interaction between this localized field and other localized fields (say, fermions) would involve overlap integrals of the form

$$
\int d z \Psi_{0}^{\dagger}(z) A(z) \Psi_{0}(z)
$$

where $\Psi_{0}$ is the fermion zero mode. We have seen that fermion zero modes may depend on various parameters (e.g., the coupling constant $h$ in the example of the previous subsection: the width of the zero mode explicitly depends on $h$, see eq. (5)). Therefore, the gauge charges in effective four-dimensional theory would be different, at least in principle, for different types of particles, and they would take arbitrary values depending on the overlap integrals like (6). This is impossible in non-Abelian gauge theories where the gauge charge is quantized, i.e., it depends only on representation to which a matter field belongs, up to a factor common to all fields.

Any mechanism of the localization of (non-Abelian) massless gauge fields must automatically preserve charge universality, i.e., ensure that gauge charges of all four-dimensional particles are the same (up to group representation factors) irrespectively of the structure 
of their wave functions in transverse directions or other details of a mechanism that binds these particles to our brane. To the best of author's knowledge, in the absence of gravity the only field-theoretic mechanism? of gauge field localization which ensures charge universality, is that of Ref. [15]. It has been proposed to consider a gauge theory which is in confinement phase in the bulk (outside the brane), whereas there is no confinement on the brane. Then the electric field of a charge residing on the brane will not penetrate into the bulk, the multi-dimensional Gauss' law will reduce to the four-dimensional Gauss' law, and the electric field on the brane will fall off according to the four-dimensional Coulomb law, $E \propto 1 / r^{2}$.

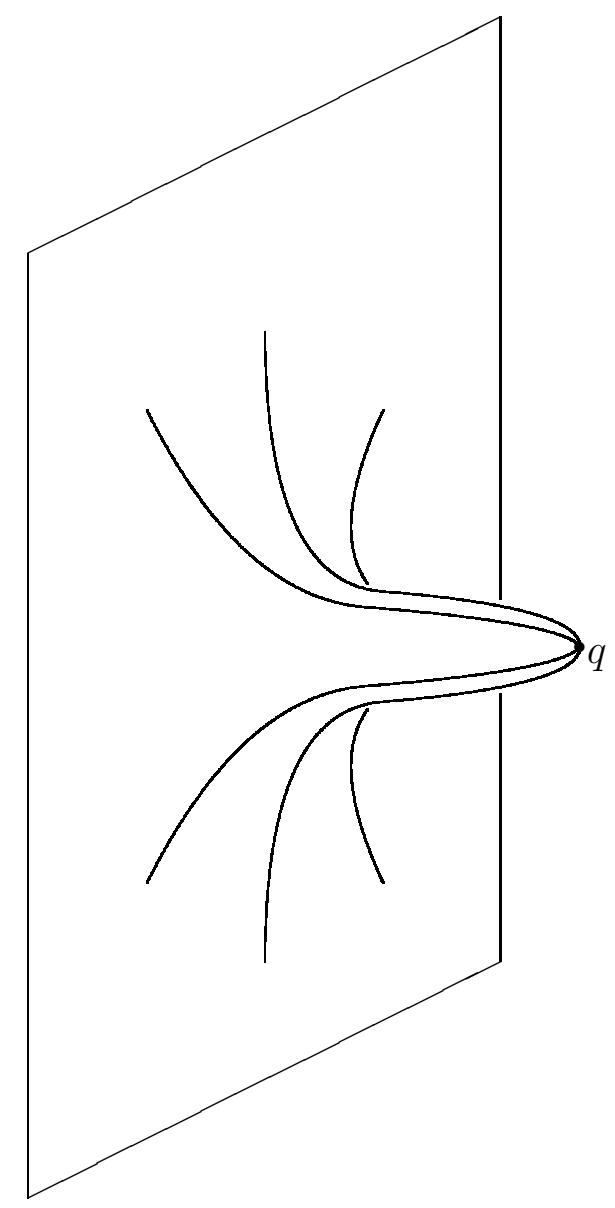

Figure 4: Charge $q$, displaced from the brane, is connected to the brane by a flux tube.

A dual analogue of this situation is an inhomogeneous superconductor with superconductivity destroyed on a plane (i.e., Cooper pair condensate vanishing on the plane). Magnetic

\footnotetext{
${ }^{1}$ If gravity is turned on, other mechanisms may appear, as we discuss later.

${ }^{2}$ An alternative mechanism proposed in Refs. [16, 17, 18] does not, in general, preserve charge universality, and, therefore, it has problems in non-Abelian case. These will be discussed elsewhere.
} 
monopoles placed far away from the plane will experience confinement (there will be an Abrikosov vortex connecting a monopole and anti-monopole), while monopoles residing on the plane will interact according to the two-dimesnional Coulomb law.

Charge universality in this setting is ensured by confinement in the bulk. If charge is displaced from the brane, a vortex connecting this charge to the brane and carrying all of the flux will be formed as shown in Fig. 4. The gauge field induced by this charge on the brane at large distances will be independent of the position of this charge in extra dimensions and will be identical to the three-dimensional Coulomb field of a charge placed exactly on the brane.

This picture can be made explicit [19] by considering an Abelian model of dual superconductivity in an arbitrary number of dimensions, along the lines of Ref. [20]. The corresponding calculations are rather involved, and we do not reproduce them here.

It is worth noting that confinement in the bulk may imply that all states propagating in the bulk are heavy. If the corresponding mass gap is large enough, light particles carrying gauge charges will be bound to the brane, and bulk modes will not be excited at low energies. Hence, the mechanism of Ref. [15] is simultaneously a mechanism of trapping matter (fermions, Higgs bosons, etc.) to the brane, alternative to that discussed in Section 3.1.

We would like to warn the reader, however, that it is not known whether non-Abelian field theories, exhibiting confinement, exist at all in more than four space-time dimensions. So, in field theory context, the mechanism of Ref. [15] in somewhat up in the air. On the other hand, the picture with confinement in the bulk and no confinement on the brane has a lot of similarities with the localization of gauge fields on $D$-branes of string/M-theory.

\section{Large extra dimensions}

\subsection{Size of extra dimensions}

Localization of matter on a brane explains why low energy physics is effectively fourdimensional insofar as all interactions except gravity are concerned. To include gravity, one may proceed in different ways. One approach [21, 22], hereafter called ADD, is to neglect the brane tension (energy density per unit three-volume of the brane) and consider compact extra dimensions. In this way the Kaluza-Klein picture is reintroduced. The size of extra dimensions $R$ need not, however, be microscopic (we assume for simplicity that the sizes of all extra dimensions are of the same order). Indeed, the distances at which non-gravitational interactions cease to be four-dimensional are determined by the dynamics on the brane, and may be much smaller than $R$. Only gravity becomes multi-dimensional at scales just below $R$. The four-dimensional law of gravitational attraction has been established experimentally down to distances[ of about $0.2 \mathrm{~mm}$ [23], so the size of extra dimensions is allowed to be as

\footnotetext{
${ }^{3}$ Until recently, the distance down to which the Newton law was established experimentally was in the several millimeter range [24, 25], for a review see Ref. [26. The new round of experiments was stimulated
} 
large as $0.1 \mathrm{~mm}$.

This possibility opens up a new way to address the hierarchy problem [21, 22], the problem of why the elctroweak scale (of order $M_{E W} \sim 1 \mathrm{TeV}$ ) is so different from the Planck scale $\left(M_{P l} \sim 10^{16} \mathrm{TeV}\right)$. In multi-dimensional theories, the four-dimensional Planck scale is not a fundamental parameter. Rather, the mass scale of multi-dimensional gravity, which we denote simly by $M$, is fundamental, as it is this latter scale that enters the full multi-dimensional gravitational action,

$$
S=-\frac{1}{16 \pi G_{(D)}} \int d^{D} X \sqrt{g^{(D)}} R^{(D)}
$$

where

$$
G_{(D)}=\frac{1}{M^{D-2}} \equiv \frac{1}{M^{d+2}}
$$

is the fundamental $D$-dimensional Newton's constant, $d=D-4$ is the number of extra dimensions, and $d^{D} X=d^{4} x d^{d} z$.

In ADD picture, the long-distance four-dimensional gravity is mediated by the graviton zero mode (cf. Section 2) whose wave function is homogeneous over extra dimensions. Hence, the four-dimensional effective action describing long-distance gravity is obtained from eq. (7) by taking the metric to be independent of extra coorinates $z$. The integration over $z$ is then trivial, and the effective four-dimensional gravitational action is

$$
S_{e f f}=\frac{V_{d}}{16 \pi G_{(D)}} \int d^{4} x \sqrt{g^{(4)}} R^{(4)}
$$

where $V_{d} \sim R^{d}$ is the volume of extra dimensions. We see that the four-dimensional Planck mass is, up to a numerical factor of order one, equal to

$$
M_{P l}=M(M R)^{\frac{d}{2}}
$$

If the size of extra dimensions is large compared to the fundamental length $M^{-1}$, the Planck mass is much larger than the fundamental gravity scale $M$.

One may push this line of reasoning to extreme and suppose that the fundamental gravity scale is of the same order as the electroweak scale, $M \sim 1 \mathrm{TeV}$. Then the hierarchy between $M_{P l}$ and $M_{E W}$ is entirely due to the large size of extra dimensions. The hierarchy problem becomes now the problem of explaining why $R$ is large. This is certainly an interesting reformulation.

Assuming that $M \sim 1 \mathrm{TeV}$, one calculates from eq. (8) the value of $R$,

$$
R \sim M^{-1}\left(\frac{M_{P l}}{M}\right)^{\frac{2}{d}} \sim 10^{\frac{32}{d}} \cdot 10^{-17} \mathrm{~cm}
$$

For one extra dimension one obtains unacceptably large value of $R$. An interesting case is $d=2$ when roughly $R \sim 1 \mathrm{~mm}$. This observation [21] stimulated recent activity in precicely by the idea that extra dimensions may be large. 
experimental search for deviations from Newton's gravity law at sub-millimeter distances. As we shall discuss later, the mass scale as low as $M \sim 1 \mathrm{TeV}$ is in fact excluded, for $d=2$, by astrophysics and cosmology; a more realistic value $M \sim 30 \mathrm{TeV}$ implies $R \sim 1-10 \mu \mathrm{m}$. This motivates search for deviations from Newton's law in a micro-meter range, which is difficult but not impossible [27, 28].

For $d>2$, eq. (9) results in smaller values of $R$. For example, for $d=3$ and $M \sim$ $1 \mathrm{TeV}$ one obtains $R \sim 10^{-6} \mathrm{~cm}$. Search for violation of Newton's law at these scales appears hopeless. For $d=6$ (full dimensionality of space time $D=10$, as suggested by superstring theory), one has $R \sim 10^{-12} \mathrm{~cm}$, which is still much larger than the elctroweak scale, $(1 \mathrm{TeV})^{-1} \sim 10^{-17} \mathrm{~cm}$. We note, however, that the compactification scales of different extra dimensions are not guaranteed to be of the same order; if some of these are much smaller than the others, the situation with deviations from Newton's gravity in spaces with $d>2$ may be similar to that of $d=2$. In other words, deviations from Newton's gravity law may occur in micro-meter range even for $d>2$.

\subsection{Light KK gravitons: colliders, cosmology and astrophysics}

If the fundamental gravity scale is indeed in the $\mathrm{TeV}$ range, one expects that extra dimensions should start to show up in collider experiments at energies approaching this scale. In the picture described in this section, extra dimensions are felt exclusively by gravitons capable of propagating in the bulk. Hence, the most distinctive feature of this scenario is the possibility to emit gravitons into the bulk; this process has strong dependence on the center-of-mass energy of particles colliding on the brane and has large probablity at energies comparable to the fundamental gravity scale.

From the four-dimensional viewpoint, emission of gravitons into extra dimensions corresponds to the production of Kaluza-Klein gravitons. One process of this type is shown in Fig. 5. Each of KK graviton states interacts with matter on the brane with four-dimensional gravitational strength. Indeed, the quadratic action for each type of KK graviton, and its interaction with matter on the brane are schematically (omitting all indices, tensor structure, etc.) written as

$$
S_{\mathbf{n}}=\frac{1}{16 \pi G_{(D)}} \int d^{D} X\left[\partial h(x) \mathrm{e}^{i \mathbf{q}_{\mathbf{n}} \mathbf{z}}\right]^{*}\left[\partial h(x) \mathrm{e}^{i \mathbf{q}_{\mathbf{n}} \mathbf{z}}\right]+\int d^{4} x h(x) T(x)
$$

where $\mathbf{q}_{\mathbf{n}}$ are the discrete momenta along extra dimensions (in the case of toroidal compactification with equal sizes of extra dimensions one has $\mathbf{q}_{\mathbf{n}}=\mathbf{n} / R, \mathbf{n}=\left(n_{1}, \ldots n_{d}\right)$ ) and $T_{\mu \nu}$ is the energy-momentum tensor of matter on the brane. The integration over $z$ again gives the volume factor $V_{(d)}$ in front of the first term, so the coupling of each type of KK graviton is determined by the four-dimensional Planck mass.

Even though the coupling of every KK graviton is weak, the total emission rate of KK gravitons is large at energies approaching $M$ due to large number of KK graviton states. The produced KK gravitons will fly away from a detector, so the typical collider processes 


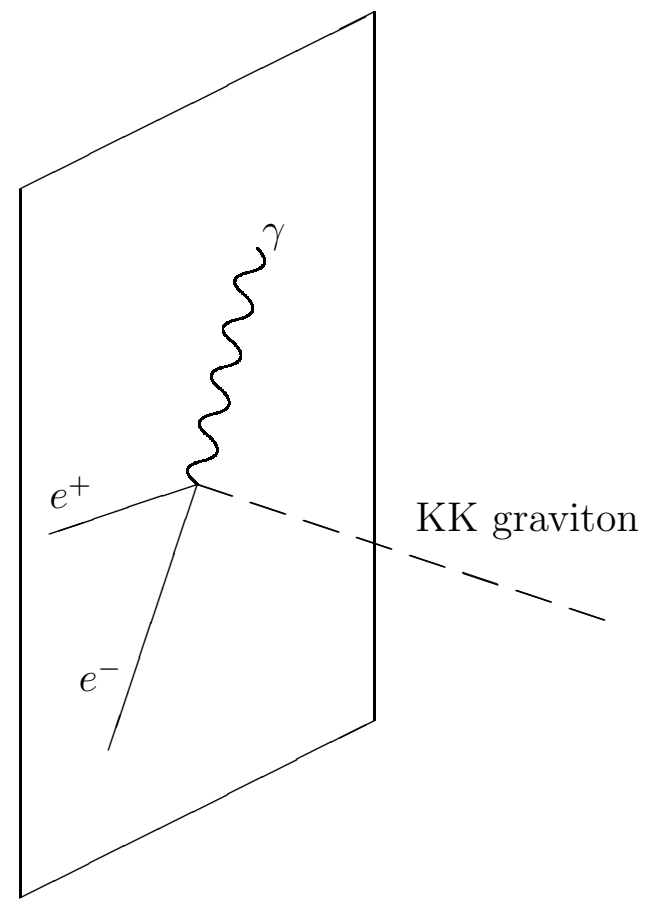

Figure 5: Emission of a graviton into extra dimensions (or, equivalently, creation of a KK graviton) in the process $e^{+} e^{-} \rightarrow \gamma+$ graviton. Electron, positron and photon propagate along the brane, the graviton escapes into the bulk.

will involve missing energy, for example

$$
e^{+} e^{-} \rightarrow \gamma+\not E_{T}
$$

or

$$
q \bar{q} \rightarrow \text { jet }+\mathbb{E}_{T}
$$

The cross section of production of a KK graviton of a given type in the process (10) is of order $\alpha / M_{P l}^{2}$, so the total cross section is of order

$$
\sigma\left(e^{+} e^{-} \rightarrow \gamma+\mathscr{E}_{T}\right) \sim \frac{\alpha}{M_{P l}^{2}} N(E)
$$

where $E$ is the center-of-mass energy, and $N(E)$ is the number of spieces of KK gravitons with masses below $E$. Since the momenta along extra dimensions, $\mathbf{q}_{\mathbf{n}}$, are quantized in units of $1 / R$, and the KK graviton masses are $m_{n}=\left|\mathbf{q}_{\mathbf{n}}\right|$, one has

$$
N(E) \sim(E R)^{d}
$$


Making use of the relation (8) one obtains

$$
\sigma\left(e^{+} e^{-} \rightarrow \gamma+\mathscr{E}_{T}\right) \sim \frac{\alpha}{E^{2}}\left(\frac{E}{M}\right)^{d+2}
$$

Hence, the cross section indeed rapidly increases with the center-of-mass energy, and at $E \sim M$ becomes comparable to the electromagnetic cross sections.

The processes (10), (111) have been analyzed in detail in Refs. [29, 30]. It has been found that CERN LHC, as well as $e^{+} e^{-}$collider with center-of-mass energy $1 \mathrm{TeV}$, will probe the fundamental gravity scale $M$ up to several $\mathrm{TeV}$, the precise number depending on $d$, the number of extra dimensions.

Another effect of extra dimensions for collider physics is the exchange of virtual KK gravitons [34, 29, 31, 32, 33]. The search for this effect at future colliders will also be sensitive to the scale $M$ in multi-TeV range.

Light KK gravitons are essentially model-independent feature of the ADD scenario (see, however, Ref.[35]). If $M \sim 1 \mathrm{TeV}$ is indeed the fundamental scale of the theory, one may expect that physics at this scale is very rich, in particular, that there exist new particles with masses of order $M$, either bound to the brane or propagating in the bulk. These particles may be fairly strongly coupled to ordinary matter; in particular, they may well carry gauge charges, and behave like heavy electrons, quarks, vector or scalar bosons. Their properties are more model-dependent, but in any case, current and future collider searches for such states are of interest from this point of view. The study of manifestations of these heavy states has been performed, in string theory context, in Refs. [36, 37, 38].

Coming back to light KK gravitons, one notes that they may have important effects in cosmology and astrophysics. In the early Universe, they can be produced at high enough temperatures, and therefore may destroy the standard Big Bang picture [34]. Consistency with the Big Bang nucleosynthesis, as well as the present composition of the Universe impose strong bounds on the maximum temperature of the Universe [34], as we shall now see.

At high enough temperature, $T \gg 1 / R$, the creation rate (per unit time per unit volume) of one KK graviton spieces of mass $m_{\mathbf{n}} \lesssim T$ is estimated as

$$
\Gamma \sim \frac{T^{6}}{M_{P l}}
$$

where the factor $M_{P l}^{-2}$ comes from the strength of the graviton-matter interaction, and the dependence on temperature is restored on dimensional grounds. Taking into account the number of KK states, cf. eq. (12), one obtains the estimate of the total rate of creation of KK gravitons,

$$
\frac{d n}{d t} \sim \frac{T^{6}}{M_{P l}}(T R)^{d} \sim T^{4}\left(\frac{T}{M}\right)^{2+d}
$$

where the latter relation comes from eq. (8). Assuming that the Universe expands in the 
standard wayt,

$$
H=\frac{T^{2}}{M_{P l}^{*}}
$$

with $M_{P l}^{*}=M_{P l} /\left(1.66 g_{*}^{1 / 2}\right) \sim($ a few $) \cdot 10^{18} \mathrm{GeV}$, where $g_{*}$ is the effective number of degrees of freedom, one finds the total number density of KK gravitons created in the Hubble time $H^{-1}$,

$$
n(T) \sim T^{2} M_{P l}^{*}\left(\frac{T}{M}\right)^{2+d}
$$

Even though the creation rate (13) is fairly small at $T \ll M$, the total number of gravitons may be large because of slow expansion rate (14).

A stringent bound on the maximum temperature $T_{*}$, that ever occurred in the Universe after inflation, emerges if one takes the ADD picture literally, i.e., assumes that KK gravitons survive in the bulk. Most of the gravitons created at temperature $T_{*}$ have masses of order $T_{*}$. Below this temperature they are non-relativistic, and their number density scales as $T^{3}$. Hence, at the nucleosynthesis epoch $\left(T_{N S} \sim 1 \mathrm{MeV}\right)$ the mass density of KK gravitons is of order

$$
\rho_{\text {grav }}\left(T_{N S}\right) \sim\left(\frac{T_{N S}}{T_{*}}\right)^{3} \cdot T_{*} n\left(T_{*}\right) \sim T_{N S}^{3} M_{P l}^{*}\left(\frac{T_{*}}{M}\right)^{2+d}
$$

Requiring that this energy density is lower than the energy density of one massless spieces (otherwise the standard Big Bang nucleosynthesis would fail), i.e., that $\rho_{\text {grav }}\left(T_{N S}\right) \lesssim T_{N S}^{4}$, one obtains

$$
T_{*} \lesssim M\left(\frac{T_{N S}}{M_{P l}^{*}}\right)^{\frac{1}{2+d}} \sim M \cdot 10^{-\frac{21}{2+d}}
$$

For $d=2$ and $M=1 \mathrm{TeV}$ one finds that the maximum temperature should not exceed $10 \mathrm{MeV}$, and even for $d=6$ one obtains fairly low maximum temperature, $T_{*} \lesssim 1 \mathrm{GeV}$.

Even stronger bounds on $T_{*}$ are obtained by requiring that the present mass density of KK gravitons does not exceed the actual energy density, which is close to the critical density, and that decaying KK gravitons do not produce too much diffuse photon background [34]. For $d=2$, the very fact that the Universe underwent the nucleosynthesis epoch (i.e., that $T_{*} \gtrsim(\mathrm{a}$ few) $\mathrm{MeV}$ ) pushes the fundamental gravity scale up to $M \gtrsim(\mathrm{a}$ few $) \cdot 10 \mathrm{TeV}$.

Low maximum temperature of the Universe (say, in the range $10 \mathrm{MeV}-1 \mathrm{GeV}$ ) does not directly contradict cosmological data: we know for sure that the Universe underwent the standard hot Big Bang evolution at the nucleosynthesis epoch, but have no observational handle on higher temperature epochs?. With low $T_{*}$, however, one has to invoke fairly exotic mechanisms of baryogenesis and inflation, which are possible but not very appealing.

We note in passing that we have assumed in the above discussion that KK gravitons do not decay before the nucleosynthesis epoch. This is correct if nothing happens to gravitons

\footnotetext{
${ }^{4}$ If KK gravitons dominated the expansion of the Universe, eq. (13) would not hold. It is straightforward to see, however, that such a scenario is not viable, so we do not consider this case.

${ }^{5}$ This has been explored in detail in Ref. [39].
} 
emitted into the bulk: the width of a graviton with mass of order $T_{*}$ with respect to decay into ordinary particles (photons, $e^{+} e^{-}$-pairs, etc.) is of order $T_{*}^{3} / M_{P l}^{2}$, which, for $T_{*} \lesssim 1 \mathrm{GeV}$ (and even for substantially larger $T_{*}$ ), is much smaller than the expansion rate before and at nucleosynthesis, $H \sim T_{N S}^{2} / M_{P l}^{*}$. One might invent mechanisms of faster decay of $\mathrm{KK}$ gravitons into something massless in the bulk (or on a different brane). Then the energy density of the latter would scale as $T^{4}$, and the cosmological constraints on $T_{*}$ would be weaker. Still, $T_{*}$ is required to be rather low. Indeed, instead of eq. (15) one would have

$$
\rho_{\text {extra }}\left(T_{N S}\right) \sim\left(\frac{T_{N S}}{T_{*}}\right)^{4} \cdot T_{*} n\left(T_{*}\right) \sim \frac{T_{N S}^{4}}{T_{*}} M_{P l}^{*}\left(\frac{T_{*}}{M}\right)^{2+d}
$$

Requiring again that $\rho_{\text {extra }}\left(T_{N S}\right) \lesssim T_{N S}^{4}$, one obtains

$$
T_{*} \lesssim M\left(\frac{M}{M_{P l}^{*}}\right)^{\frac{1}{2+d}}
$$

For $M \sim 1 \mathrm{TeV}$ and $d=2$ one again obtains $T_{*} \lesssim 10 \mathrm{MeV}$, whereas for $d=6$ one has $T_{*} \lesssim 10 \mathrm{GeV}$. This model-independent estimate shows that the maximum temperature must be rather low irrespectively of the fate of emitted KK gravitons.

Light KK gravitons are potentially dangerous for astrophysics as well, as they may be produced by stars or supernovae, take away energy, and hence contradict observational data [34]. Strong bounds on the fundamental scale $M$ are obtained in this way for $d=2$ only, as for larger number of extra dimensions, the number of KK graviton states with small masses is suppressed, cf. eq. (12). As an example, by requiring that the emission of gravitons during the collapse of SN1987a is not the dominant cooling process (otherwise no neutrinos would be produced, in contradiction to observations), one obtains [34, 40, 41, 42]

$$
M>30 \mathrm{TeV}
$$

which is comparable to the cosmological bounds. Note, however that this bound is obtained without any assumptions concerning the lifetime of KK gravitons. As we alredy mentioned, with so high scale $M$, the deviations from Newton's law are only allowed well below $1 \mathrm{~mm}$ - more realistically, at distances at most in 1 to $10 \mu \mathrm{m}$ range. Even stronger bound is obtained in Ref. [43] under the assumption that KK gravitons produced during suprenovae collapses decay into usual photons (and not, say, into particles residing on other branes).

Thus, the ADD picture predicts interesting phenomena at TeV energy scale: its modelindependent feature is the existence of light KK gravitons which would show up at colliders either directly, in processes (10), (11), or indirectly, through contact interactions induced by the exchange by virtual KK gravitons. Model-dependent features include heavy partners of ordinary particles. Cosmology in the ADD scenario is not, however, very appealing: the maximum temperature of the Universe must be below $10 \mathrm{GeV}$, so one has to rely upon fairly exotic mechanisms of baryogenesis and inflation. 


\subsection{Sterile neutrinos in the bulk}

Besides gravitons, there may exist other light fields not bound to the brane and freely propagating in the bulk. The large number of their KK states of very small masses will be phenomenologically acceptable only if these fields are neutral under the Standard Model gauge group $S U(3)_{c} \times S U(2)_{L} \times U(1)_{Y}$. An interesting candidate is a neutral fermion which couples to conventional left-handed neutrinos and the Standard Model Higgs field, both trapped to the brane. This interaction induces naturally small Dirac neutrino masses 44, 45.

The $D$-dimensional action involving $D$-dimensional fields $\Psi_{\nu}$ and $H$, whose zero modes describe four-dimensional flavor neutrino and Higgs boson, respectively, and a neutral bulk fermion $\Psi$ is

$$
S_{(D)}=\int d^{4} x d^{d} z \bar{\Psi} \Gamma^{A} \partial_{A} \Psi-\kappa \int d^{4} x d^{d} z \bar{\Psi}_{\nu} H \Psi+\text { h.c. }+\ldots
$$

where dots denote terms without the neutral fermion $\Psi$. The coupling constant has dimension (mass) $)^{-d / 2}$. Let us consider the effective four-dimensional theory of the usual neutrino and Higgs field interacting with KK modes of the bulk fermion. For $\Psi_{\nu}$ and $H$ we write

$$
\begin{aligned}
\Psi_{\nu}(x, z) & =\nu(x) \psi_{0}(z) \\
H(x, z) & =h(x) H_{0}(z)
\end{aligned}
$$

where $\nu(x)$ and $H(x)$ are four-dimensional fields and $\psi_{0}(z)$ and $H_{0}(z)$ are wave functions in transverse dimensions. The latter concentrate near the brane, $z=0$, and are normalized to unity. For the neutral bulk fermion, we have KK decomposition

$$
\Psi(x, z) \propto \sum_{\mathbf{n}=0}^{\infty} \psi_{\mathbf{n}}(x) \frac{1}{R^{\frac{d}{2}}} \mathrm{e}^{i \mathbf{n z} / R}
$$

where the factor $R^{-d / 2}$ is introduced for canonical normalization of the four-dimensional fermion $\psi_{n}(x)$. The effective four-dimensional theory is then described by the effective action

$$
S_{e f f}=\sum_{\mathbf{n}=0}^{\infty} \int d^{4} x\left(\bar{\psi}_{\mathbf{n}} \gamma^{\mu} \partial_{\mu} \psi_{\mathbf{n}}-m_{\mathbf{n}} \bar{\psi}_{\mathbf{n}} \psi_{\mathbf{n}}-\frac{\kappa^{\prime}}{R^{\frac{d}{2}}} \bar{\nu}_{L} h \psi_{\mathbf{n}, R}\right)+\ldots
$$

where $m_{\mathbf{n}} \sim|\mathbf{n}| / R$ and we assume that the neutrino zero mode is four-dimensionally lefthanded (which is necessary for realistic phenomenology and is natural in field-theoretic models, as discussed in Section 3). The coupling $\kappa^{\prime}$ involves the overlap integral of $\psi_{0}(z)$ and $H_{0}(z)$ which does not contain $R$-dependent factors, and hence is naturally of order one, so that

$$
\kappa^{\prime} \sim \kappa
$$

Once the Higgs field $h$ acquires vacuum expectation value $v \sim M_{E W}$, the last term in eq.(18) induces the Dirac neutrino mass

$$
m_{\nu, L R}=\frac{\kappa^{\prime} v}{R^{\frac{d}{2}}}
$$


For $n=0$ (the lowest KK state of the bulk fermion), this is the only mass term in the effective theory; this implies that if $m_{\nu, L R} \ll 1 / R$ (i.e., $m_{\nu, L R} \ll m_{\mathbf{n}}$ for $\mathbf{n} \neq 0$ ), then the $\mathrm{KK}$ modes with $\mathbf{n} \neq 0$ are irrelevant at very low energies, and the four-dimensional theory reduces to the theory of a Dirac neutrino with mass (19), plus other sterile fermions which decouple.

Since the Dirac mass (19) is suppressed by the size of extra dimensions, it is naturally small. Taking, as a crude estimate,

$$
\kappa^{\prime} \sim \kappa \sim M^{-\frac{d}{2}}
$$

where $M$ is again the fundamental gravity scale, one finds

$$
m_{\nu, L R} \sim \frac{v}{(M R)^{\frac{d}{2}}}
$$

Recalling the relation (8), one obtains

$$
m_{\nu, L R} \sim \frac{v M}{M_{P l}}
$$

which is of order $10^{-4} \mathrm{eV}$ for $M \sim$ (a few). TeV. This estimate suggests that the neutrino masses may naturally fall in the ballpark suggested by solar and atmospheric neutrino data.

It is worth noting that the reason for smallness of the neutrino masses in this picture has precisely the same origin as the smallness of the four-dimensional Newton's constant: the bulk fields spread over the whole space of extra dimensions, and thus interact very weakly with matter residing on the brane.

Refinement of this picture has lead to a number of interesting effects 45, 46, 47, 48, 49, 50. The Yukawa coupling in eq. (16) is not, in general, flavour-diagonal. This property, and especially mixing of flavor neutrinos $\nu_{e}, \nu_{\mu}, \nu_{\tau}$ with higher $\mathrm{KK}$ states $\psi_{n}$ induce neutrino oscillation patterns which are often very non-trivial. It has been found that in a reasonably wide region of the parameter space, these patterns are consistent with experimental data on neutrino oscillations, and that this mechanism can be discriminated, in future experiments, against conventional four-dimensional mechanisms. There are, however, strong constraints on this scenario, notably, coming from SN 1987a [48, 49]. These and other issues are reviewed, e.g., in Ref. [51].

\subsection{Unification of couplings}

A nice property of the four-dimensional Minimal Supersymmetric Standard Model (MSSM), and many of its extensions, is that the gauge couplings $\alpha_{i}, i=1,2,3$, corresponding to the gauge groups $U(1)_{Y}, S U(2)_{L}$ and $S U(3)_{c}$, respectively, unify at the Grand Unification scale $M_{G U T} \sim 10^{16} \mathrm{GeV}\left(\alpha_{1}\right.$ is actually defined as $\left.(5 / 3) \alpha_{Y}\right)$. This occurs through the logarithmic running of these couplings according to the renormalization group. Gauge coupling unification is a very strong argument in favor of both MSSM and Grand Unification. 
In theories with large extra dimensions, this argument is apparently lost, as gravity becomes strong at relatively low energies, so completely new physics (strings) is to set in many orders of magnitude below $M_{G U T}$.

The situation is not so hopeless, however. There have been discussed at least two possibilities to obtain gauge coupling unification in theories with large extra dimensions. One of them exploits power-law running of couplings in higher-dimensional theories [52], another invokes massless fields propagating in two large transverse dimensions [53, 54, 55] and leads to logarithmic unification.

The idea of power-law unification is as follows. Suppose that there exists an energy scale $\mu_{0}$ at which extra dimensions open up for gauge, Higgs and possibly quark and lepton fields of MSSM. In other words, suppose that MSSM particles can leave our brane provided they have energy exceeding $\mu_{0}$. Then below this scale, MSSM is effectively four-dimensional, whereas above this scale it is $D$-dimensional. In four-dimensional language, each MSSM particle has its KK partners whose masses start at $\mu_{0}$. The scale fundamental gravity scale $M$ should then be considered as an ultraviolet cut-off for the $D$-dimensional MSSM. Since MSSM is non-renormalizable in more than four dimensions, the low energy values of the gauge couplings depend strongly (as a power law) on $M$ and $\mu_{0}$. In this sense the gauge couplings exhibit power-law running at scales above $\mu_{0}$. The question is whether there exist $M$ and $\mu_{0}$, both very roughly in the TeV range, such that the gauge coulings unify at the cut-off scale,

$$
\alpha_{1}^{-1}(M)=\alpha_{2}^{-1}(M)=\alpha_{3}^{-1}(M) \equiv \alpha_{G U T^{\prime}}
$$

and yet their low energy values are equal to the experimentally measured ones,

$$
\begin{aligned}
& \alpha_{Y}^{-1}\left(M_{Z}\right)=98.3, \quad \text { i.e. }, \alpha_{1}^{-1}\left(M_{Z}\right)=\frac{3}{5} \alpha_{Y}\left(M_{Z}\right)=59.0 \\
& \alpha_{2}^{-1}\left(M_{Z}\right)=29.6 \\
& \alpha_{3}^{-1}\left(M_{Z}\right)=8.5
\end{aligned}
$$

The relation (20) would then substitute the conventional unification of couplings and suggest that there is a Grand Unified Theory above the fundamental scale $M$. It is the power-law running of couplings that makes this possibility not inconceivable.

Let us consider a theory in $d$ flat extra dimensions compactified on a torus, as we did before in this Section. Consistent embedding of MSSM in a higher-dimensional theory requires additional particles above the scale $\mu_{0}$. A minimal extension [52 is that the KK tower has effective $N=2$ supersymmetry: from the four-dimensional viewpoint, there is $N=2$ vector supermultiplet for each gauge group, an $N=2$ hypermultiplet for the two Higgs fields and $\eta$ families of $N=2$ hypermultiplets of quarks and leptons. With this matter content, the one-loop relation between the low energy values of the gauge couplings and their values at the cut-off is [52]

$$
\alpha_{i}^{-1}(M)=\alpha_{i}^{-1}\left(M_{Z}\right)-\frac{b_{i}}{2 \pi} \ln \frac{M}{M_{Z}}+\frac{\tilde{b}_{i}}{2 \pi} \ln \frac{M}{\mu_{0}}-\frac{\tilde{b}_{i} \pi^{\frac{d}{2}-1}}{d^{2} \Gamma\left(\frac{d}{2}\right)}\left[\left(\frac{M}{\mu_{0}}\right)^{d}-1\right]
$$


where $\left(b_{1}, b_{2}, b_{3}\right)=(33 / 5,1,-3)$ are the usual MSSM one-loop $\beta$-function coefficients, and

$$
\left(\tilde{b}_{1}, \tilde{b}_{2}, \tilde{b}_{3}\right)=\left(\frac{3}{5}+4 \eta,-3+4 \eta,-6+4 \eta\right)
$$

are " $\beta$-function coefficients" of higher-dimensional theory. The power-law dependence of $\left[\alpha_{i}^{-1}\left(M_{Z}\right)-\alpha_{i}^{-1}(M)\right]$ on $M$ and $\mu_{0}$, which is evident from eq. (22), is just the power-law running of couplings; it reflects their dimensionality in $D=d+4$ dimensions.

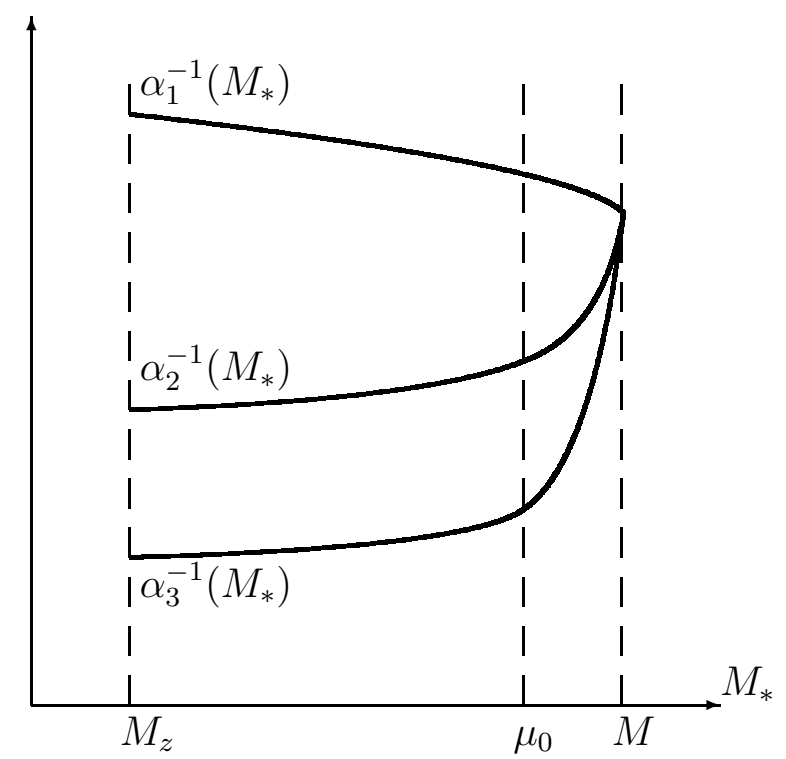

Figure 6: Schematic plot of the unification of gauge couplings in the presence of extra dimensions. For fixed $\alpha_{i}\left(M_{z}\right)$, the gauge couplings at the cut-off scale $M_{*}$ depend on this scale (logarithmically at $M_{*}<\mu_{0}$ and as power law at $M_{*}>\mu_{0}$ ). Unification occurs at $M_{*}=M$, where $M$ is interpreted as the fundamental scale.

Somewhat miraculously, the unification of couplings indeed takes place [52], as shown schematically in Fig. 6. This occurs irrespectively of the number of extra dimensions, $d$, and the number of bulk quark-lepton generations, $\eta$. Namely, for given $M$ (and fixed $d$ and $\eta)$, the parameter $\mu_{0}$ can be chosen in such a way that eq. (20) is satisfied. Like in the fourdimensional MSSM, this property is non-trivial in higher-dimensional theories: by choosing one parameter $\mu_{0}$ one satisfies two equations simultaneously. The unification occurs for $\mu_{0}$ just (within about an order of magnitude) below $M$ - the couplings run very fast above $\mu_{0}$. The gauge coupling at the unification point is safely smaller than unity,

$$
\alpha_{i}(M) \equiv \alpha_{G U T^{\prime}} \ll 1
$$

It has been argued in Ref. [52 that this picture is unchanged when higher loops are taken into account. Thus, contrary to the naive expectation, the property of coupling constant 
unification may be inherent in theories with large extra dimensions, although instead of logarithmic unification, one may have faster, power-law unification in these theories.

It is worth noting that in theories with large extra dimensions, unification of gauge couplings does not, generally speaking, require supersymmetry. In a number of non-supersymmetric higher-dimensional extensions of the Standard Model, gauge couplings exhibit power-law unification as well [52]. Hence, both motivations for supersymmetry - stabilization of the electroweak scale and gauge coupling unification - are not as strong in theories with large extra dimensions as they are in four-dimensional theories.

The power-law unification has its problems, however. A technical problem is that this picture is generically unstable against the effects of possible non-renormalizable operators added at the cut-off scale. Aesthetically, it is not appealing that the "old" unification is given up: the logarithmic unification inherent in MSSM appears a pure accident.

The logarithmic "running" of gauge couplings in theories with large extra dimensions may occur in the following, rather unexpected way [53, 54, 55]. Suppose, in the spirit of string theory, that the gauge couplings are in fact massless scalar fields $\alpha_{i}^{-1}(z)$ which propagate in two large extra dimensions?. Suppose also that besides our brane, there exists another brane which serves as a source for these fields, and at which the couplings are unified,

$$
\alpha_{i}^{-1}\left(z_{\text {source }}\right)=\alpha_{0}^{-1}, \quad i=1,2,3
$$

Naturally, the distance between our and source branes is of the order of the size of extra dimensions $R$. Since the two-dimensional propagator is a logarithm, the values of $\alpha_{i}^{-1}(z)$ at the position of our brane are?

$$
\alpha_{i}^{-1}\left(z_{\text {our }}\right)=\alpha_{0}^{-1}-\frac{c_{i}}{2 \pi} \ln \left(\left|z_{\text {our }}-z_{\text {source }}\right| M\right)
$$

with some constants $c_{i}$, where the fundamental scale $M$ appears in the logarithm on dimensional grounds. For $\left|z_{\text {our }}-z_{\text {source }}\right| \sim R \sim M_{p l} / M^{2}$ (as given by eq. (8) with $d=2$ ) the logarithmic term here is

$$
-\frac{c_{i}}{2 \pi} \ln \left(\frac{M_{P l}}{M}\right)
$$

which is almost the same logarithm as $\ln \left(\frac{M_{G U T}}{M_{E W}}\right)$ that occurs in conventional Grand Unification.

Unlike conventional renormalization group evolution, "running" described by eq. (23) is an infrared effect. Still, it works in right direction: the values of gauge couplings in our world differ from the "fundamental" value $\alpha_{0}^{-1}$ by large logarithms. The most miraculous thing is that in certain stringy brane constructions, constants in eq. (23) are precisely the $\beta$-function coefficients of the theory localizad on our brane! The discussion of this property and its relation to string dualities goes far beyond this mini-review; the reader

\footnotetext{
${ }^{6}$ The actual number of large extra dimensions need not be equal to two; it is important only that the scalar fields are effectively two-dimensional [55].

${ }^{7}$ Here, the energy scale for $\alpha_{i}^{-1}\left(z_{\text {our }}\right)$ and $\alpha_{0}^{-1}$ is the fundamental scale $M$.
} 
may consult Ref. [55] and references therein. We stress only that this property opens up a way towards gauge coupling unification, not merely logarithmic "running" of couplings. Although a phenomenologically acceptable model with MSSM on our brane has not been constructed along these lines yet, this mechanism is certainly promising, as it suggests that the logarithmic unification existing in MSSM may have its close counetrpart in theories with large extra dimensions.

\subsection{Problem with proton stability}

The above discussion brings us to one more problem with large (and also infinite) extra dimensions - potentially too fast proton decay. This problem is apparent if one takes the attitude of the previous subsection and considers the unification of gauge couplings as a signal of Grand Unification of strong and electroweak interactions at the scale $M$. In conventional Grand Unified Theories, dimension six operators lead to proton decay with life-time of order $\$$

$$
\tau_{p} \sim \frac{1}{\alpha_{G U T}^{2} M_{p}}\left(\frac{M_{G U T}}{M_{p}}\right)^{4}
$$

With $M_{G U T} \sim 10^{16} \mathrm{GeV}$, this is consistent with experimental limits on the proton instability, but if $M_{G U T}$ is shifted to $M$ (which is roughly in the TeV range in ADD scenario discussed in this Section), this estimate gives way too small proton life-time. Grand Unification at the scale $M$ should somehow occur in such a way that the proton width is suppressed by many orders of magnitude, as compared to the dimensional estimate.

More generally, global quantum numbers, such as baryon and lepton number, may not be conserved when quantum gravity effects are included. With fundamental gravity scale $M_{P l} \sim 10^{19} \mathrm{GeV}$, dimensional estimate similar to eq. (25), i.e.,

$$
\tau_{p} \sim \frac{1}{M_{p}}\left(\frac{M_{P l}}{M_{p}}\right)^{4}
$$

shows that proton instability due to gravity effects is not phenomenologically dangerous. However, if the fundamental gravity scale is in the $\mathrm{TeV}$ range, the same estimate gives unacceptably small proton life-time. Hence, one has to invoke special mechanisms (such as discrete gauge symmetries [56, 57, 58, 59, 60]) to forbid proton decay in theories with large extra dimensions.

\section{Non-factorizable geometry}

\footnotetext{
${ }^{8}$ In supersymmetric GUTs, proton decay occurs also due to dimension five operators. We consider the contribution (25) merely as an illustration.
} 


\subsection{Warped extra dimension}

Until now we have ignored the energy density of the brane itself, i.e., the gravitational field that the brane produces. Here we shall see that a gravitating brane induces an interesting geometry in multi-dimensional space, and that a number of novel properties emerge.

When considering distance scales much larger than the brane thickness, one may view the brane as a delta-function source of the gravitational field. In the simplest case, the gravitating brane is characterized by just one parameter, the energy density per unit threevolume $\sigma$. This quantity is also called brane tension. We shall mostly discuss the case of one extra dimension, so the five-dimensional gravitational action in the presence of the brane is

$$
S_{g}=-\frac{1}{16 \pi G_{(5)}} \int d^{4} x d z \sqrt{g^{(5)}} R^{(5)}-\Lambda \int d^{4} x d z \sqrt{g^{(5)}}-\sigma \int d^{4} x \sqrt{g^{(4)}}
$$

where $\Lambda$ is the five-dimensional cosmological constant, and the integral in the last term is evaluated along the world surface of the 3 -brane with $g_{\mu \nu}^{(4)}$ being the induced metric.

The resulting field equations are straightforward to obtain. In the bulk, these are the standard five-dimensional Einstein equations with the cosmological constant $\Lambda$, while the last term in eq. (26) gives rise to the Israel junction conditions [61] on the brane surface (for pedagogical presentation of the Israel conditions see, e.g., Ref. [62]). Notably, this set of equations allows for a solution preserving four-dimensional Poincaré invariance. This fact was extensively discussed in the $D$-brane context (see, e.g, Ref. [63] and references therein) and its relevance for phenomenological models has been stressed in Refs. [64, 65, 66, 67]. The existence of four-dimensionally flat solution requires fine-tuning between $\Lambda$ and $\sigma$ : the five-dimensional cosmological constant must be negative and equal to 66]

$$
\Lambda=-\frac{4 \pi}{3} G_{(5)} \sigma^{2}
$$

(note that the parameters here have dimensions $[\Lambda]=M^{5},[\sigma]=M^{4},\left[G_{(5)}\right]=M^{-3}$ ). This fine-tuning is very similar to fine-tuning of the cosmological constant to zero in conventional four-dimensional gravity; indeed, if eq. (27) does not hold, the intrinsic geometry on the brane is (anti-)de Sitter rather than flat.

With the relation (27) satisfied, the four-dimensionally flat solution has the form 66]

$$
d s^{2}=a^{2}(z) \eta_{\mu \nu} d x^{\mu} d x^{\nu}-d z^{2}
$$

where $\eta_{\mu \nu}$ is the four-dimensional Minkowski metric and the "warp factor" has the form

$$
a(z)=\mathrm{e}^{-k|z|}
$$

where

$$
k=\frac{4 \pi}{3} G_{(5)} \sigma
$$

The brane is located at $z=0$. 
To see that this is indeed a solution to the complete system of the Einstein equations, we write for the metric (28)

$$
\begin{aligned}
G_{\mu \nu} \equiv R_{\mu \nu}^{(5)}-\frac{1}{2} g_{\mu \nu}^{(5)} R^{(5)} & =g_{\mu \nu}^{(5)}\left[-3 \frac{a^{\prime \prime}}{a}-3\left(\frac{a^{\prime}}{a}\right)^{2}\right] \\
G_{z \mu} & =0 \\
G_{z z} & =g_{z z}^{(5)}\left[-6\left(\frac{a^{\prime}}{a}\right)^{2}\right]
\end{aligned}
$$

where prime denotes the derivative with respect to $z$. In is then straightforward to see that metric (28) is a solution to the Einstein equations

$$
\begin{aligned}
G_{\mu \nu} & =8 \pi G_{(5)} \Lambda g_{\mu \nu}^{(5)}+8 \pi G_{(5)} \sigma g_{\mu \nu}^{(5)} \delta(z) \\
G_{z \mu} & =0 \\
G_{z z} & =8 \pi G_{(5)} \Lambda g_{z z}^{(5)}
\end{aligned}
$$

provided eq. (29) is satisfied, and

$$
k^{2}=-\frac{4 \pi}{3} G_{(5)} \Lambda
$$

which is equivalent to eq. (27). Note that eq. (29) comes from the requirement

$$
-3 \frac{\left[a^{\prime}\right]}{a} g_{\mu \nu}^{(5)}=8 \pi G_{(5)} \sigma g_{\mu \nu}^{(5)}, \quad z=0
$$

where $\left[a^{\prime}\right]$ denotes the jump of $a^{\prime}$ at $z=0$. The latter requirement is essentially the Israel condition in the case considered.

The metric (28) is non-factorizable: unlike the metrics appearing in the usual KaluzaKlein scenarios, it does not correspond to a product of the four-dimensional Minkowski space and a (compact) manifold of extra dimensions. This metric rather corresponds to two patches of anti-de Sitter space of radius $1 / k$ glued together along $z=0$, i.e., along the brane. The four-dimensional hypersurfaces $z=$ const are flat; in particular, the metric induced on the brane is the Minkowski metric $\eta_{\mu \nu}$.

At this point it is worth mentioning one property of the metric (28). Due to fourdimensional Poincaré invariance, every field in this background can be decomposed into four-dimensional plane waves,

$$
\phi \propto \mathrm{e}^{i p_{\mu} x^{\mu}} \phi_{p}(z)
$$

The coordinate four-momentum $p_{\mu}$ coincides with the physical momentum on the brane, but from the point of view of an observer residing at $z \neq 0$, the physical four-momentum is larger,

$$
p_{\mu}^{p h y s}(z)=\frac{1}{a(z)} p_{\mu}=\mathrm{e}^{k|z|} p_{\mu}
$$

The modes which are soft on the brane become harder away from the brane. This scaling property is behind many peculiarities of physics in the background (28). 


\subsection{Two-brane set up}

There are several approaches which make use of the solution (28). One of them [66] is to make extra dimension compact by introducing two branes: one with positive tension $\sigma$ at $z=0$, and the other with negative tension $(-\sigma)$ located at distance $z_{c}$, see Fig. 7. Allowing the negative tension brane to vibrate freely is dangerous, as this will give rise to physical excitations of arbitrarily large negative energy (see Ref. 68 for detailed discussion). To circumvent this problem, the branes are placed at fixed points of an orbifold; in our case, this means that all bosonic fields, including gravity, are required to be symmetric under reflections with respect to both $z_{c}$, the position of the negative tension brane, and $z=0$, the position of positive tension one (fermion fields may have more complicated symmetry properties). The metric (28) is still a solution of the complete set of Einstein equations in the presence of the two branes; extra dimension is compact, as the coordinate $z$ runs now from $z=0$ to $z=z_{c}$,

$$
z \in\left[0, z_{c}\right]
$$

The orbifold boundary conditions (reflection symmetry) project the undesirable negative energy modes out, and there remain positive energy excitations only.

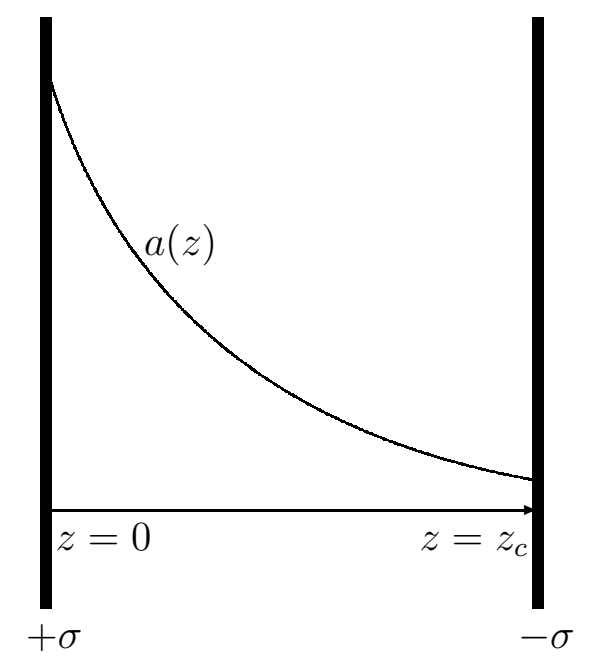

Figure 7: Two-brane set up: branes of positive and negative tensions and warp factor between them.

Let us consider small perturbations about the metric (28). To make the long story short, one can use the gauge

$$
g_{55}=-1, \quad g_{5 \mu}=0
$$

i.e., consider perturbed metric of the form

$$
d s^{2}=\left[a^{2}(z) \eta_{\mu \nu}+h_{\mu \nu}(x, z)\right] d x^{\mu} d x^{\nu}-d z^{2}
$$


If there are no sources of the gravitational field except for the bulk cosmological constant and the two branes, one can further specify the coordinate frame (i.e., fix the gauge), so that $h_{\mu \nu}$ are transverse and trace-free in the bulk,

$$
\partial_{\mu} h_{\nu}^{\mu}=0, \quad h_{\mu}^{\mu}=0
$$

For all types of perturbations but one, this frame is at the same time Gaussian normal with respect to both branes. This means that the positions of the branes are still $z=0$ and $z=z_{c}$. Then all components of $h_{\mu \nu}$ obey the same equation (we omit the subscripts)

$$
h^{\prime \prime}-4 k^{2} h-\frac{m^{2}}{a^{2}(z)} h=0
$$

where

$$
m^{2}=\eta^{\mu \nu} p_{\mu} p_{\nu}
$$

is the four-dimensional mass of the perturbation. The junction conditions on the branes are (assuming the orbifold symmetry)

$$
h^{\prime}+2 k h=0 \quad \text { at } z=0 \text { and } z=z_{c}
$$

Equations (33) and (34) determine the mass spectrum of the KK gravitons, where the mass is defined with respect to the positive tension brane, cf. eq. (32).

Before considering this spectrum, let us point out that there exists one scalar mode which cannot be treated in the above way. This mode - the radion — is massless and corresponds to oscillations of the relative distance between the two branes. Its properties are discussed, e.g., in Refs. [69, 70, 68]. In many phenomenological models based on this set up, massless radion is unacceptable (we shall briefly discuss this point below). Giving the radion a mass corresponds to stabilizing the distance between the branes; field theory mechanisms for this stabilization is suggested, e.g., in Ref. [71, 72]. We shall not consider the radion mode in what follows, assuming that the distance between the branes is stabilized in one or another way.

Let us now turn to the graviton spectrum, i.e., solutions to eqs (33) and (34). There exists a zero mode, $m^{2}=0$, whose wave function, up to normalization, is

$$
h_{0}(z)=\mathrm{e}^{-k z}
$$

This mode describes the usual four-dimensional gravity. Unlike in the Kaluza-Klein theories with factorizable geometry, the zero-mode wave function depends on $z$ non-trivially, and decreases towards $z=z_{c}$. This suggests that the gravitational coupling between particles residing on the negative tension brane is weak as compared to the positive tension brane. We shall discuss this feature in more detail later on.

Solutions to eq. (33) obeying the boundary condition (34) at $z=0$ (not yet at $z=z_{c}$ ) are, again up to normalization,

$$
h_{m}(z)=N_{1}\left(\frac{m}{k}\right) J_{2}\left(\frac{m}{k} \mathrm{e}^{k z}\right)-J_{1}\left(\frac{m}{k}\right) N_{2}\left(\frac{m}{k} \mathrm{e}^{k z}\right)
$$


where $N$ and $J$ are the Bessel functions. The mass spectrum is determined by the boundary condition (34) at $z=z_{c}$. One obtains that the mass splitting between the KK modes is of order

$$
\Delta m \sim k \mathrm{e}^{-k z_{c}}
$$

The phenomenological interpretation of these results depends on whether the Standard Model particles are bound to the brane of positive or negative tension.

\subsection{Matter on negative tension brane and hierarchy}

Let us first consider the possibility that the ordinary matter resides on the negative tension brane (RS1 scenario [66]). We are interested in gravitational interactions of this matter at large distances; then the dominant contribution to the gravitational attraction is due to the zero graviton mode. It is convenient to rescale the four-dimensional coordinates in such a way that the warp factor is equal to 1 at the negative tension brane (i.e., at $z=z_{c}$ ),

$$
a(z)=\mathrm{e}^{k\left(z_{c}-z\right)}
$$

Similarly, it is convenient to normalize the zero mode so that it is equal to 1 at the negative tension brane. Then the massless gravitational perturbations are described by the field

$$
h_{\mu \nu}(x, z)=\mathrm{e}^{2 k\left(z_{c}-z\right)} h_{\mu \nu}^{(4)}(x)
$$

The coordinates $x^{\mu}$ are now the physical coordinates on the negative tension brane, and the four-dimensional graviton field $h_{\mu \nu}^{(4)}(x)$ couples to energy-momentum of the ordinary matter in the usual way,

$$
S_{i n t}=\int d^{4} x h_{\mu \nu}^{(4)} T^{\mu \nu}
$$

The strength of gravitational interactions is read off from the quadratic part of the action for $h_{\mu \nu}^{(4)}$. This is obtained by plugging the expression (38) in the five-dimensional gravitational action (26). Schematically, one has

$$
\begin{aligned}
S_{g} & =\frac{1}{16 \pi G_{(5)}} \int_{-z_{c}}^{z_{c}} \frac{d z}{a^{2}(z)} d^{4} x\left(\partial_{\mu} h\right)^{2} \\
& =\frac{1}{8 \pi G_{(5)}} \int_{0}^{z_{c}} d z \mathrm{e}^{2 k\left(z_{c}-z\right)} \int d^{4} x\left(\partial_{\mu} h^{(4)}\right)^{2} \\
& =\frac{\left(\mathrm{e}^{2 k z_{c}}-1\right)}{16 \pi G_{(5)} k} \int d^{4} x\left(\partial_{\mu} h^{(4)}\right)^{2}
\end{aligned}
$$

(the factor $a^{-2}$ in the first integral can be either obtained directly, or inferred from the structure of eq. (33)). The integral in the last expression is the quadratic action for fourdimensional gravitons. Hence, the four-dimensional Newton's constant is

$$
G_{(4)}=G_{(5)} k \frac{1}{\mathrm{e}^{2 k z_{c}}-1}
$$


which means that at relatively large $z_{c}$, the gravitational interactions of matter residing on the negative tension brane are weak.

This observation opens up a novel possibility to address the hierarchy problem. Indeed, one can take the fundamental five-dimensional gravity scale, as well as the inverse anti-de Sitter radius $k$ to be of the order of the weak scale, $M_{E W} \sim 1 \mathrm{TeV}$. As is clear from eq. (41), the effective four-dimensional Planck mass is then of order

$$
M_{P l} \sim \mathrm{e}^{k z} M_{E W}
$$

which means the exponential hierarchy between the Planck mass and the weak scale: for $z_{c}$ only about 37 times larger than the anti-de Sitter radius $k^{-1}$, the value of $M_{P l} / M_{E W}$ is of the right order of magnitude.

One may wonder whether the low energy theory of gravity obtained in this set up is indeed the conventional four-dimensional General Relativity. It has been shown explicitly in Refs. [73, 74] that this is indeed the case, provided the radion is given a mass (see also [69]). Otherwise the radion would act as a Brans-Dicke field with unacceptably strong ( TeV scale) coupling to matter.

Let us now turn to KK gravitons. The mass splitting (37) refers to the masses measured by an observer on the positive tension brane. According to eq. (32), the physical masses measured by an observer on the negative tension brane are of order

$$
m_{\text {grav }} \sim k
$$

Hence, KK gravitons have masses in $\mathrm{TeV}$ range, in clear distinction to ADD scenario. In particular, the cosmological difficulties inherent in ADD picture do not appear here: the maximum temperature of the Universe is allowed to be just below the TeV range.

Unlike the zero mode, the coupling of KK gravitons to matter residing on the negative tension brane is characterized by the fundamental mass scale (of order $M_{E W}$ ). To see this, we write the massive modes as follows (for $m$ somewhat larger than $k \mathrm{e}^{-k z_{c}}$ )

$$
h_{m}(x, z)=\mathrm{e}^{k\left(z_{c}-z\right) / 2} \sin \left(\frac{m}{k} \mathrm{e}^{k z}-\varphi_{m}\right) h_{m}^{(4)}(x)
$$

This expression is valid at $(m / k) \mathrm{e}^{k z} \gg 1$, while the KK wave functions decrease towards $z=0$. The pre-factor in eq. (43) has been chosen in such a way that the four-dimensional fields $h_{m}^{(4)}(x)$ couple to matter at $z=z_{c}$ with unit strength, cf. eq. (39). In the same way as eq. (40) one obtains the quadratic action

$$
\begin{aligned}
S_{g, m} & =\frac{1}{16 \pi G_{(5)}} \int \frac{d z}{a^{2}(z)} d^{4} x\left(\partial_{\mu} h_{m}\right)^{2} \\
& =\frac{1}{8 \pi G_{(5)}} \int_{0}^{z_{c}} d z \mathrm{e}^{-k\left(z_{c}-z\right)} \int d^{4} x\left(\partial_{\mu} h_{m}^{(4)}\right)^{2} \\
& =\frac{\left(1-\mathrm{e}^{-k z_{c}}\right)}{8 \pi G_{(5)} k} \int d^{4} x\left(\partial_{\mu} h_{m}^{(4)}\right)^{2}
\end{aligned}
$$


Hence, the mass scale determining the interactions of KK gravitons with matter is of order

$$
M_{m} \sim \frac{1}{\sqrt{G_{(5)} k}}
$$

which is of order $M_{E W}$. The interaction of matter and KK gravitons becomes strong in the TeV energy range.

Thus, RS1 scenario leads to exponential hierarchy between the weak and Planck scales. Similarly to ADD, gravity becomes strong at TeV energies; manifestations of this phenomenon in collider experiments will be quite different in RS1 model as compared to ADD [75. The reason is of course that the graviton spectra are entirely different; a distinctive feature of RS1 collider phenomenology is TeV scale graviton resonances quite strongly coupled to ordinary particles. For further discussion of RS1 phenomenology see Refs. [76] and references therein.

\subsection{Matter on positive tension brane}

Another option is that the conventional matter resides on the positive tension brane. The analysis similar to that leading to eq. (41) shows that in this case the effective fourdimensional Newton's constant is

$$
G_{(4)}=G_{(5)} k \frac{1}{1-\mathrm{e}^{-2 k z_{c}}}
$$

If one does not introduce huge hierarchy between the fundamental five-dimensional gravity scale and the inverse anti-de Sitter radius $k$, the fundamental scale must be of order of $M_{P l}$. This does not mean, however, that the exponential hierarchy between the scales cannot be generated. There is a possibility that the electroweak symmetry breaking and/or supersymmetry breaking occur due to physics on the negative tension brane, and are transfered to "our" brane by one or another mechanism [77. In that case the electroweak scale and/or supersymmetry breaking scale in our world are naturally exponentially smaller than the Planck scale, essentially because of the scaling relation (32), and the exponential hierarchy (42) is again generated. Concerete models of this sort have been discussed in Ref. [77], and their phenomenology turned out to be quite interesting. Note that the masses of KK gravitons are again in the $\mathrm{TeV}$ range: these are given by eq. (37) with $k \sim M_{P l}$.

\section{Infinite extra dimension}

\subsection{Localized graviton}

The graviton zero mode (35) that appeared in the set up of Section 5, is normalizable for $z_{c} \rightarrow \infty$, i.e., for negative tension brane moved away. This means that gravity is still localized if there exists a single positive tension brane only, and extra dimension is infinite 
[67. Hence, one is lead to consider a set up, called RS2 [67, with matter residing on the positive tension brane and experiencing four-dimensional gravity law at large distances due to the exchange of the graviton zero mode. The fact that gravity is four-dimensional at large distances is clear also from eq. (45): in the limit $z_{c} \rightarrow \infty$, the four-dimensional Newton's constant tends to a finite value

$$
G_{(4)}=G_{(5)} k
$$

Obviously, in this simplest set up, unlike in constructions of Sections 4 and 5, the hierarchy between the Planck and weak scales is not explained by physics of extra dimension, and one has to rely upon other, more conventional mechanisms (we shall mention another option later on). The interest in this simplest set up with infinite extra dimension is, on the one hand, due to potentially interesting physics at low energies, and, on the other hand, due to its connection to adS/CFT correspondence (for a brief review of adS/CFT see, e.g., Ref. [78]).

One property of the anti-de Sitter geometry in the bulk is worth mentioning. Although the distance from the brane to $z=\infty$, measured along the $z$-axis is infinite, it is straightforward to see that $z=\infty$ is in fact a particle horizon. Indeed, let us consider, as an example, a massive particle that starts from the brane at $t=0$ and $\mathbf{x}=0$ with zero velocity, and then freely moves along the $z$-axis. The corresponding solution to the geodesic equation is 179, 80,

$$
z_{c}(t)=\frac{1}{2 k} \ln \left(1+k^{2} t^{2}\right)
$$

The particle accelerates towards $z \rightarrow \infty$, its velocity tends to the speed of light. According to eq. (28), the proper-time interval is determined by

$$
d \tau^{2}=a^{2}\left(z_{c}(t)\right) d t^{2}-\left(\frac{d z_{c}}{d t}\right)^{2} d t^{2}
$$

The particle reaches $z=\infty$ at infinite time $t$, but finite proper time

$$
\tau=\int_{0}^{\infty} \frac{d t}{1+k^{2} t^{2}}=\frac{\pi}{2 k}
$$

Hence, $z=\infty$ is indeed the particle horizon. When considering physics in the background (28), one has to impose certain boundary conditions at the horizon $z=\infty$ (which in principle may affect physics on the brane); it is usually assumed that nothing comes in from "behind the horizon".

To substantiate the claim that gravity experienced by matter residing on the (positive tension) brane is effectively four-dimensional at large distances, let us consider the KaluzaKlein gravitons. According to eq. (36), the spectrum of KK gravitons is continuous and starts from zero $\mathrm{m}^{2}$. In this situation, the wave functions of KK gravitons are to be normalized to delta-function (again with the measure $a^{-2} d z$, see eq. (40)),

$$
\int \frac{d z}{a^{2}(z)} h_{m}(z) h_{m^{\prime}}(z)=\delta\left(m-m^{\prime}\right)
$$


Making use of the asymptotics of the Bessel functions, one obtains that the properly normalized KK graviton wave functions are

$$
h_{m}(z)=\sqrt{\frac{m}{k}} \frac{J_{1}\left(\frac{m}{k}\right) N_{2}\left(\frac{m}{k} \mathrm{e}^{k z}\right)-N_{1}\left(\frac{m}{k}\right) J_{2}\left(\frac{m}{k} \mathrm{e}^{k z}\right)}{\sqrt{\left[J_{1}\left(\frac{m}{k}\right)\right]^{2}+\left[N_{1}\left(\frac{m}{k}\right)\right]^{2}}}
$$

At large $z$, these wave functions oscillate,

$$
h_{m}(z)=\text { const } \cdot \sin \left(\frac{m}{k} \mathrm{e}^{k z}+\varphi_{m}\right)
$$

whereas they decrease towards small $z$ and are suppressed at $z=0$,

$$
h_{m}(0)=\text { const } \cdot \sqrt{\frac{m}{k}}
$$

The wave functions (46) correspond to gravitons escaping into extra dimension, i.e., towards $z \rightarrow \infty$ (or coming towards the brane from $z=\infty$ ). The coupling of these KK gravitons to matter, residing on the brane, is fairly weak at small $m$, so their production at relatively low energies (and/or temperatures) is unimportant (for details, see Ref. 82] and references therein). Likewise, the contribution of virtual KK gravitons into low energy processes is small.

As an example, let us consider the contribution of KK graviton exchange into gravitational potential between two unit point masses placed on the brane. Each KK graviton produces the potential of Yukawa type, so the total contribution is

$$
\begin{aligned}
\Delta V_{K K}(r) & =-G_{(5)} \int_{0}^{\infty} d m\left|h_{m}(0)\right|^{2} \frac{\mathrm{e}^{-m r}}{r} \\
& =-\frac{G_{(5)} k}{r} \cdot \text { const } \cdot \int_{0}^{\infty} \frac{m d m}{k^{2}} \mathrm{e}^{-m r} \\
& =-\frac{G_{(4)}}{r} \cdot \frac{\text { const }}{k^{2} r^{2}}
\end{aligned}
$$

Hence, the gravitational potential, including the contribution of the graviton zero mode, is 67

$$
V(r)=-\frac{G_{(4)}}{r}\left(1+\frac{\text { const }}{k^{2} r^{2}}\right)
$$

The correction to Newton's law has power law behaviour at large $r$, in contrast to theories with compact extra dimensions where the corrections are suppressed exponentially at large distances. However, this correction is negligible at distances exceeding the anti-de Sitter radius $k^{-1}$. It has been explicitly shown in Refs. [73, 74] that the tensor structure of the gravitational interactions at large distances indeed corresponds to (the weak field limit of) the four-dimensional General Relativity. Note that the radion excitation is absent in RS2 set up. 
We have already mentioned that in RS2 set up with one brane, extra dimension does not help to solve the hierarchy problem. It was pointed out, however, that modest extension of this set up leads to exponential hierarchy even if extra dimension is infinite [81. Instead of assuming that our matter is bound to the "central" brane, one may introduce one more, "probe" brane which is placed at a position $z_{c}$ in extra dimension and, for simplicity, has zero tension. Metric (28) on this probe brane still has four-dimensional Poincaré invariance. If our matter is put on the probe brane, the exponential hierarchy (42) is generated in much the same way as in RS1 set up discussed in Section 5 .

\subsection{Escape into extra dimension}

If one or more extra dimensions are infinite, one naturally expects that particles may eventually leave our brane and escape into extra dimension. In RS2 set up, this process is certainly possible for gravitons, as the excitation of a KK mode is interpreted precisely as escape of a graviton towards $z \rightarrow \infty$. If other fields have bulk modes, the corresponding particles may also leave our brane. As an example, even in the absence of gravity, fermions bound to the brane by the mechanism presented in subsection 3.1, are capable of leaving the brane provided they are given enough energy. As we discussed in subsection 3.1, this would show up as a process like $e^{+} e^{-} \rightarrow$ nothing which would be possible at high energies.

A novelty of the bulk with anti-de Sitter metric is that such processes become possible also at low energies 83. The ultimate reason is again the scaling property (32): energies which are low if measured at the brane position, become high if measured at large $z$. Low energy physics on the brane is high energy physics away from the brane.

Quantitatively, this feature is manifest in a peculiar property of KK continuum for fields having bulk modes: the continuum starts from zero $\mathrm{m}^{2}$ irrespectively of the dynamics near the brane. Suppose now, that in the absence of gravity, a field has a bound state of a non-zero mass, whose wave function concentrates near the brane and hence corresponds to a four-dimensional particle. When gravity is turned on, this would-be bound state becomes embedded in a continuum of KK modes, which describe particles capable of escaping to $z \rightarrow \infty$. Hence, this would-be bound state becomes quasi-localized (there are no true bound states embedded in continuum, unless the potential is very contrived): its energy obtains an imaginary part which determines (finite) probability of tunneling to large $z$. Particle on the brane becomes metastable against escape into extra dimension.

To illustrate this fairly general phenomenon, let us consider real scalar field in the presence of the brane, with the action

$$
S_{\phi}=\int d^{4} x d z \sqrt{g}\left[\frac{1}{2} g^{A B} \partial_{A} \phi \partial_{B} \phi-\frac{1}{2} V(z) \phi^{2}\right]
$$

where $x^{A}=\left(x^{\mu}, z\right)$ are coordinates in five-dimensional space-time. Effects of the brane are encoded in the potential $V(z)$, which is assumed to tend to a (possibly, non-zero) nonnegative constant as $z \rightarrow \infty$ (we asuume the orbifold symmetry $z \rightarrow-z$ for simplicity). If 
gravity is switched off, the field $\phi$ obeys the Klein-Gordon equation

$$
-\partial_{\mu}^{2} \phi+\partial_{z}^{2} \phi-V(z) \phi=0
$$

The spectrum of four-dimensional masses is determined by the potential $V(z)$,

$$
p_{\mu} p^{\mu} \phi \equiv m^{2} \phi=\left[-\partial_{z}^{2}+V(z)\right] \phi
$$

An interesting case is when the operator on the right hand side of this equation has discrete eigenmodes which correspond to particles trapped to the brane. This situation is shown in Fig. 8. The continuum starts at $m^{2}=V(\infty)$.

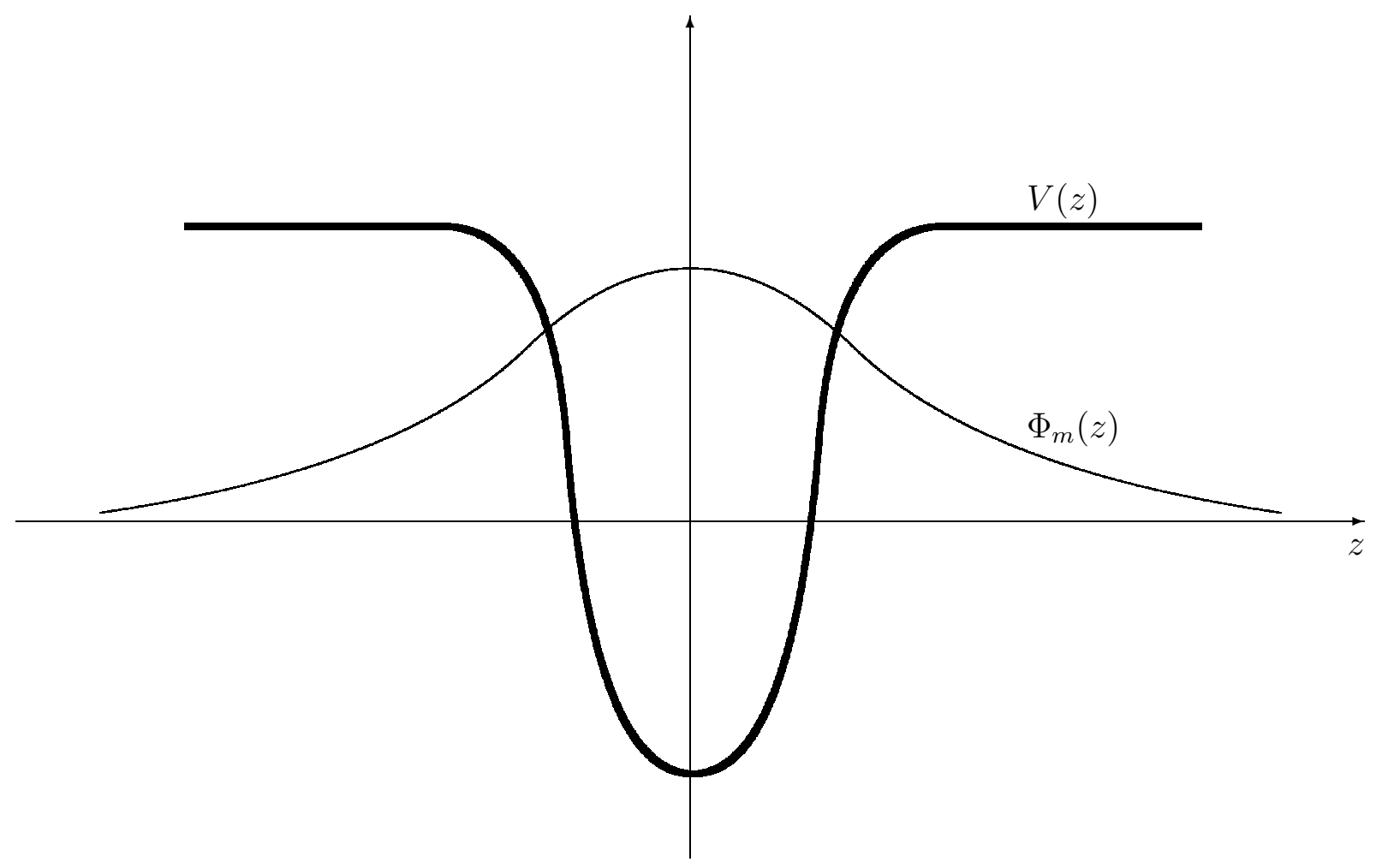

Figure 8: Binding potential $V(z)$ and bound state with $m^{2} \neq 0$ in the absence of the warp factor.

When gravity of the brane is turned on, the situation changes. The action in the background metric (28) is

$$
S_{\phi}=\int d^{4} x d z a^{4}\left[\frac{1}{a^{2}} \eta^{\mu \nu} \partial_{\mu} \phi \partial_{\nu} \phi-\frac{1}{2}\left(\partial_{z} \phi\right)^{2}-\frac{1}{2} V(z) \phi^{2}\right]
$$


where, as before, $a(z)=\mathrm{e}^{-k|z|}$. Since $a^{-2}$ grows at large $z$, the first term in the integrand of eq. (50) dominates away from the brane over the potential term, and continuum of KK modes starts from zero. The eigenvalue equation for four-dimensional masses reads now

$$
\frac{1}{a^{4}} \partial_{z}\left(a^{4} \partial_{z} \phi\right)-V(z) \phi+\frac{m^{2}}{a^{2}} \phi=0
$$

It is useful to note that the normalization condition for the eigenfunctions $\phi_{m}(z)$ is

$$
\int d z a^{2}(z) \phi_{m}(z) \phi_{m^{\prime}}(z)=\delta_{m m^{\prime}}
$$

Indeed, the eigenvalue equation (51) can be written in the form

$$
-\partial_{z}\left(a^{4} \partial_{z} \phi_{m}\right)+a^{4} V(z) \phi_{m}=m^{2} a^{2} \phi_{m}
$$

The operator entering the left hand side is Hermitean, so the eigenfunctions are orthogonal with the measure $a^{2} d z$. This is precisely the same measure as multiplies the kinetic term in the action, i.e., the first term in the integrand of eq. (50).

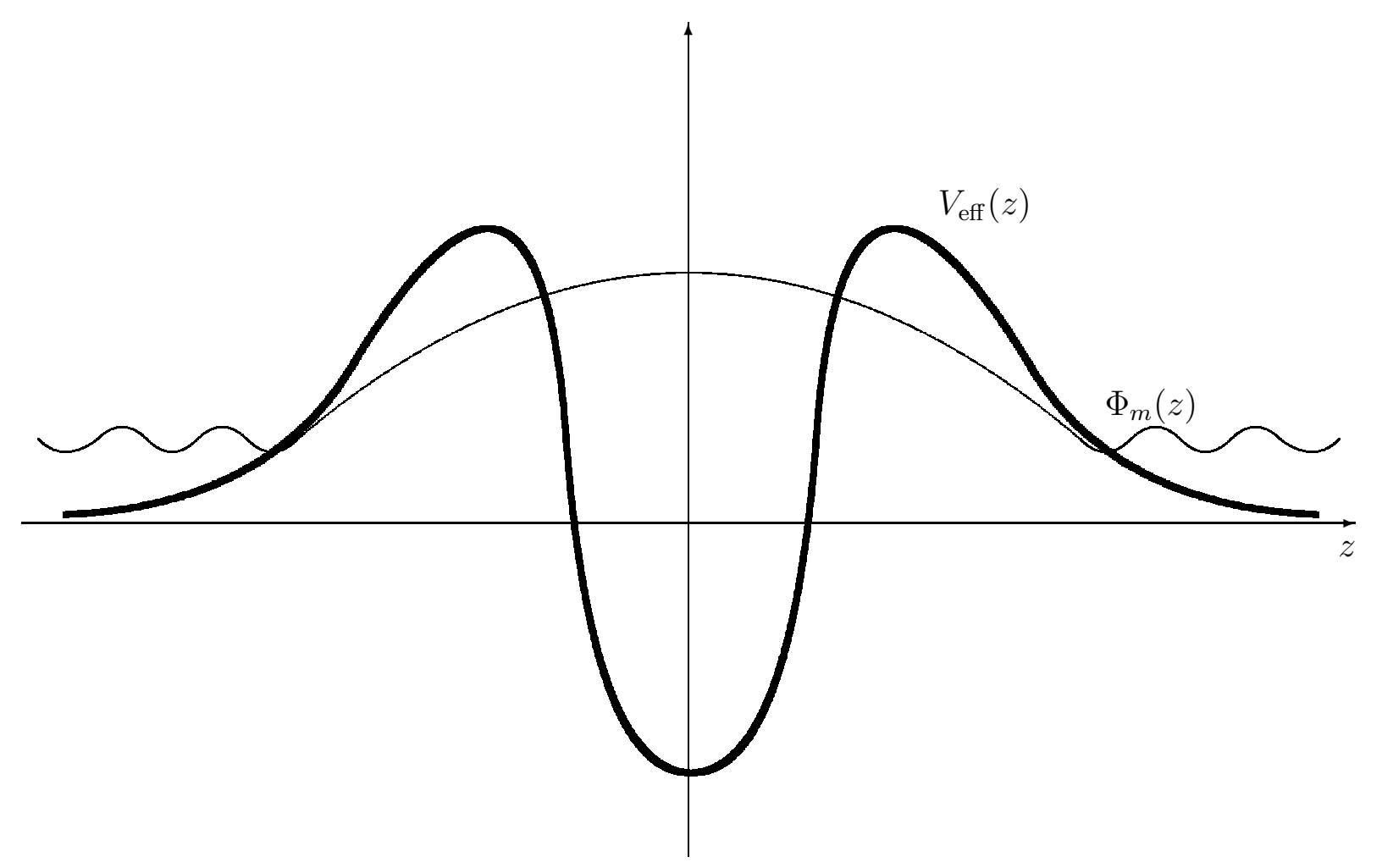

Figure 9: In anti-de Sitter space, the effective binding potential gets modified, and would-be bound state with $m^{2} \neq 0$ becomes quasi-localized. Particle has finite probability to escape the brane via tunneling. 
At large $z$, the second term in eq. (51) is negligible as compared to the third one. Effectively, this means that the binding potential gets modified and tends to zero as $|z| \rightarrow \infty$, see Fig. 9. The wave functions at $z \rightarrow \infty$ are

$$
\phi(z)=\text { const } \cdot \mathrm{e}^{3 k z / 2} \sin \left(\frac{m}{k} \mathrm{e}^{k z}+\varphi_{m}\right)
$$

(these are normalized to delta-function with weight $a^{2} d z$ ). The point is that the continuum spectrum is determined by the large- $z$ asymptotics of eq. (51), and it starts from $m^{2}=0$ irrespectively of the form of $V(z)$ (provided that $V(z)$ does not rapidly increase as $z \rightarrow \infty$ ). As there are no bound states embedded in the continuum, the massive bound states of eq. (49) become now resonances, i.e., quasi-localized states having finite widths of decay (finite probability of escape from the brane to $z=\infty$ ). These widths depend on the potential $V(z)$ binding the particles to the brane.

It is worth noting that in RS2 set up, massless scalar field has a zero mode even without the potential $V(z)$ 84]. Its wave function is $\phi=$ const, and it is normalizable with the appropriate weight $a^{2} d z$. Once this field is given a mass, the would-be bound state becomes metastable against escape into extra dimension. For example, for constant $V(z) \equiv \mu^{2}$, the mass of the four-dimensional particle is equal to 83

$$
m=\frac{\mu}{\sqrt{2}}
$$

and its width of escape to $z=\infty$ is

$$
\Gamma=m \frac{\pi}{16}\left(\frac{m}{k}\right)^{2}
$$

The latter formula illustrates a general feature of these decays: for small mass of the wouldbe bound state, the probability of its decay into extra dimension is small, the reason being that the decay occurs through tunneling, and hence it is naturally suppressed.

The above general arguments imply that the metastability of massive particles against escape into extra dimension should be characteristic to all kinds of matter, including fermions, provided these have bulk modes. The calculation of the life-time of a fermion bound to the brane by the mechanism of subsection 3.1, has been performed in Ref. 83. This life-time depends not only on the fermion mass and anti-de Sitter radius, but also on other parameters, so quantitative estimates are premature at this stage.

Yet another interesting property of anti-de Sitter bulk has to do with virtual KK states. With massive four-dimensional bosons only, the potential between sources is of the Yukawa type. Once there exist arbitrarily light KK states, one expects the potential to have long ranged tail. For example, in the scalar field model (50) with constant $V(z) \equiv \mu^{2}$, one finds the potential between two distant sources of the scalar field, $q_{1}$ and $q_{2}$, located on the brane 83

$$
V(r)=-\pi q_{1} q_{2} k \frac{\mathrm{e}^{-m r}}{r}-60 \pi q_{1} q_{2} \cdot \frac{1}{k m^{4}} \cdot \frac{1}{r^{7}}
$$


where $m$ is given by eq. (53). The first (Yukawa) term here is due to the exchange by massive quasi-localized mode, whereas the second one is due to the exchange by the KK continuum states. In a model meant to describe a massive four-dimensional particle, the potential has power-law behaviour at large distances! The assumption that the field has bulk modes, which has been crucial for the above discussion, is not, in fact, an innocent one, especially if particles are charged. Indeed, if gauge fields are localized by the mechanism of subsection 3.2 , charged particles are confined in the bulk, so they do not have bulk modes. In this scenario, escape of a charged particle from the brane into extra dimension is impossible. Later on we shall describe another mechanism of the gauge field localization, which will allow for a charged particle to escape to $z \rightarrow \infty$.

\subsection{Holographic interpretation}

Is it possible to describe the low energy physics on the brane entirely in four-dimensional language? Certainly not, if one thinks in terms of usual weakly coupled theories allowing for particle interpretation: continuum KK modes do not correspond to particles travelling in four-dimensional space-time along the brane. On the basis of adS/CFT correspondence [85, 86, 87] it has been argued 88, 89, however, that RS2 scenario may be described by a strongly coupled four-dimensional conformal field theory (CFT) with an ultraviolet cut-off, interacting with conventional gravitational field. The correction (47) to Newton's gravity law is then interpreted as coming from "one loop" contribution of conformal matter to the graviton propagator [88, 89, 76]. Indeed, in conformal field theory language, this correction to the graviton propagator has the following form (indices are omitted),

$$
\delta G(x-y)=\text { const } \int d^{4} u d^{4} v D(x-u)\langle T(u) T(v)\rangle D(v-y)
$$

where $D(x-y)$ is a free four-dimensional graviton propagator, and $T(u)$ is the energymomentum tensor of conformal fields. Hence,

$$
\square_{x} \square_{y}(\delta G(x-y))=\mathrm{const} \cdot\langle T(x) T(y)\rangle
$$

Now, the correlator of the energy-momentum tensor of a conformal field theory is

$$
\langle T(x) T(y)\rangle=\frac{\text { const }}{(x-y)^{8}}
$$

so that

$$
\delta G(x-y)=\frac{\text { const }}{(x-y)^{4}}
$$

The contribution to the gravitational potential is the time integral of this expression, which immediately gives

$$
\Delta V(r)=\frac{\text { const }}{r^{3}}
$$


in agreement with eq. (47). This is the most straightforward check of the interpretation of KK gravitons in terms of four-dimensional conformal field theory; there are a number of other checks.

Likewise, escape of a particle into extra dimension has a CFT interpretation as a decay into conformal modes, now interacting with matter fields, whereas the power-law correction to the Yukawa potential, eq. (54), is again due to the exchange by these modes.

Another way to see how effective conformal matter shows up in four-dimensional theory is to consider gravitational field of a massive point-like particle which sits on the brane until some moment of time (say, $t=0$ ) and then leaves the brane and escapes into extra dimension along the geodesic normal to the brane [80, 90]. The four-dimensional gravitational field induced by this particle is straightforward to calculate in linearized five-dimensional theory. One finds that outside the light cone, i.e., at $\left(\mathrm{x}^{2}-t^{2}\right)>0$, the four-dimensional gravitational field induced on the brane is still described by the linearized Schwarzschild metric (in other words, four-dimensional gravitational field does not change outside the light cone, in accord with causality). Inside the light cone, the induced four-dimensional metric is flat. If one defines the effective four-dimensional energy-momentum tensor by

$$
8 \pi G_{(4)} T_{\mu \nu}^{(e f f)} \equiv R_{\mu \nu}^{(4)}-\frac{1}{2} g_{\mu \nu}^{(4)} R^{(4)}
$$

where $g_{\mu \nu}^{(4)}$ is the four-dimensional metric induced on the brane, then $T_{\mu \nu}^{(e f f)}$ corresponds to a thin shell of matter expanding along the four-dimensional light cone and dissipating as $1 / r^{2}$. This is precisely the behaviour of energy-momentum expected in any conformal field theory 91 .

The holographic approach is useful for analysing aspects of phenomenology of RS2 model [74, 90, 76 and sheds new light on cosmology with infinite extra dimesnions (see, e.g., Refs. [92, 93, 94] and references therein). Conformal field theory language is useful, to a certain extent, also in RS1 scenario with two branes and compact extra dimension (see, e.g., Ref. [76] and references therein). In that case, the four-dimensional interpretation is based on strongly coupled theory with broken conformal invariance.

These examples show that four-dimesional conformal field theories, weakly coupled to ordinary matter, may emerge naturally in various multi-dimensional contexts. Irrespectively of extra dimensions, it is of interest to understand phenomenological implications of possible existence in nature of a conformal sector with broken or unbroken conformal invariance.

\subsection{More than one extra dimensions. Localization of gauge fields by gravity}

One approach to extend the construction outlined above to space-time of more than one extra dimension is to consider intersecting branes of codimension one [95]. In $(3+n)$ dimensional space, each of these branes has dimesnion $(3+n-1)$, so none of them by itself is a candidate for our world. However, an intersection of $(n-1)$ of these branes is a three-dimensional manifold, to which our matter may be bound. Furthermore, gravity is 
naturally localized on this intersection manifold, so this set up is phenomenologically viable 95.

Localizing gravity on a genuine three-brane embedded in space with more than one extra dimensions is more difficult. For many solutions of the Einstein equations in space-time of more than one transverse dimensions, the gravitational effect of the brane vanishes at large distances. To some extent, the situation here is similar to classical solutions in electrodynamics: while the electric field of a charged plane extends to infinity, the electric fields of a charged line and a charged point decay as $1 / r$ and $1 / r^{2}$, respectively. Similarly, with more than one extra dimensions, the gravitational field of a massive brane often decreases towards infinity in transverse space, so there is no reason for the localization of a graviton on the brane.

One interesting exception is the geometry with compact, but warped, additional dimen$\operatorname{sion}(\mathrm{s})$. In the case of two extra dimensions, metric of such a set up, away from the brane is

$$
d s^{2}=a^{2}(z)\left[\eta_{\mu \nu} d x^{\mu} d x^{\nu}-d \theta^{2}\right]-d z^{2}
$$

where $z$ is the coordinate along a single non-compact extra dimension, $\theta$ is a compact extra coordinate running from 0 to $2 \pi R$ and the warp factor is still

$$
a(z)=\mathrm{e}^{-k|z|}
$$

This metric is an symptotics of a solution to the $(4+1+1)$-dimensional Einstein equations with negative bulk cosmological constant and a three-brane with appropriately tuned energymomentum tensor [96] (see Refs. [97, 98] for related solutions). The brane itself is a sort of cosmic string. The generalizations of this construction to more than two extra dimensions have been found in Ref. [99, 100].

Another way to obtain metrics of the type (55) is to consider a $(3+n)$-brane embedded in $(3+n+1)$-dimensional space [101]. In other words, one considers a brane of codimension one, as in RS2 set up, but now in $(5+n)$-dimensional space-time. Then the metric

$$
d s^{2}=a^{2}(z)\left[\eta_{\mu \nu} d x^{\mu} d x^{\nu}-\delta_{i j} d \theta^{i} d \theta^{j}\right]-d z^{2}
$$

which is a straightforward generalization of eq. (28), obeys the full Einstein equations with essentially the same fine-tuning conditions as in the original RS2 set up. One then takes $\theta^{i}$ to be compact coordinates, $\theta^{i} \in\left(0,2 \pi R_{i}\right)$, so that these dimensions are invisible at low energies in complete analogy to the Kaluza-Klein picture.

In either case, there exists a graviton zero mode which is independent of $\theta^{i}$ outside the brane and decreases at large $z$ as $\mathrm{e}^{-2 k|z|}$. Gravity is localized on the brane in the same way as in RS2 case.

A novel feature of this set up is the localization of gauge fields by gravity [102, 101]. With appropriate normalization, the action of the gauge field in the background (56) is

$$
S_{\text {gauge }}=-\frac{1}{4} \int \prod_{i} \frac{d \theta^{i}}{2 \pi R_{i}} d z d^{4} x \sqrt{g} g^{A C} g^{B D} F_{A B} F_{C D}
$$


At low energies, $E \ll 1 / R_{i}$, the relevant gauge field configurations are independent of $\theta^{i}$. We shall be interested in four-vector part of the gauge field, so we choose the gauge $A_{z}=0$ and set $A_{\theta^{i}}=0$. Then the linearized gauge field equations are

$$
\begin{aligned}
-\frac{1}{a^{2+n}} \partial_{z}\left(a^{2+n} \partial_{z} A_{\mu}\right)+\frac{1}{a^{2}} \eta^{\lambda \nu} \partial_{\lambda} F_{\nu \mu} & =0 \\
\partial_{z}\left(\eta^{\mu \nu} \partial_{\mu} A_{\nu}\right) & =0
\end{aligned}
$$

In complete analogy to eq. (52), the eigenfunctions of eq. (57) are to be normalized with the measure

$$
\int d z a^{n}
$$

For $n>0$ (one or more compact warped dimensions), there exists a zero mode, which is independent of $z$,

$$
A_{\mu}=A_{\mu}(x)
$$

This mode is normalizable with the measure (59) and corresponds to massless vector boson localized on the brane.

The fact that the zero mode of the gauge field is constant over extra dimensions, ensures charge universality, which otherwise would be an obstacle for the gauge boson localization, as discussed in subsection 3.2. With constant gauge boson zero mode, the overlap integrals like eq. (6) become the norms of matter zero modes, whose values do not depend on the shapes of the zero modes. This way to get around the charge universality problem relies, of course, entirely on special geometry in the bulk.

Besides the zero mode, gauge fields in the background (56) have arbitrarily light bulk modes; the gauge theory is not in confinement phase in the bulk. This is in sharp contrast to the mechanism discussed in subsection 3.2. Furthermore, the gravitational mechanism of the gauge field localization allows the electric charge, and other gauge charges, to leak into extra dimensions. This would mean effective non-conservation of gauge charges in our world [101]. A process " $e^{-} \rightarrow$ nothing" would be a clear signature of infinite extra dimensions.

A problem [103 with the set up with warped compact dimension(s) is that the metric (56) has a singularity at $z=\infty$. Indeed, the sizes of compact dimensions, $\mathrm{e}^{-k|z|} R_{i}$, tend to zero as $z \rightarrow \infty$. Although the proper distance to the singularity along the $z$-axis is infinite, proper distance along time-like geodesics is finite. This implies that physics near the singularity may affect physics on the brane. Possible resolutions of this singularity and their effects on physics on the brane have been discussed recently in Ref [103.

\section{$7 \quad$ Further developments}

Both versions of the brane world picture - with large and flat extra dimensions, and with warped extra dimensions - have been developed along many different lines. Without pretending to give an account of any degree of completeness, let us briefly discuss a few of them. 


\subsection{Cosmological constant}

In the brane world context, the cosmological constant problem may be reformulated as a problem of why the vacuum energy density has (almost) no effect on the curvature induced on our brane. This reformulation sounds suggestive, as it implies that the vacuum energy density may affect the overall geometry of multi-dimensional space-time, but this may occur in such a way that metric induced on our brane is (almost) flat. Off hand, it seems plausible that, in the case of non-factorizable geometry, the vacuum energy density may induce a non-trivial warp factor, while the four-dimensional Poincaré invariance remains intact. The latter possibility may exist irrespectively of the brane world picture [104, 105].

Recent attempts [106, 107] to solve the cosmological constant problem in the framework of brane world and warped extra dimension, combine these ideas with the suggestion, put forward in a similar context in four-dimensional theories [108, 109, 110, that a hypothetic scalar field conformally coupled to our matter may play an important role. This field is taken to be a bulk field, so the action is a sum of the bulk and brane contributions,

$$
S=S_{b u l k}+S_{\text {brane }}
$$

Here the bulk term is

$$
S_{b u l k}=\int d z d^{4} x \sqrt{g^{(5)}}\left(-\frac{1}{2 \kappa_{(5)}} R^{(5)}+\frac{b}{2} g^{A B} \partial_{A} \phi \partial_{B} \phi\right)
$$

where $\kappa_{(5)}=8 \pi G_{(5)}, b$ is an arbitrary constant and space-time is five-dimensional. The brane contribution is assumed to have the following structure,

$$
S_{\text {brane }}=\int d^{4} x \sqrt{g^{(4)} \mathrm{e}^{4 \kappa_{(5)} \phi(0)}} \mathcal{L}\left(\psi, g_{\mu \nu}^{(4)} \mathrm{e}^{\kappa_{(5)} \phi(0)}\right)
$$

where $\mathcal{L}$ is the complete Lagrangian of matter on the brane, $\psi$ stands for all fields on the brane, $g_{\mu \nu}^{(4)}$ is the induced metric and $\phi(0) \equiv \phi(x, z=0)$ is the induced scalar field. In the absence of real particles on the brane, the brane Lagrangian reduces to the vacuum energy, with all quantum effects included, interacting with gravitational and scalar fields

$$
S_{\text {brane }}=-\epsilon_{\text {vac }} \int d^{4} x \sqrt{g^{(4)} \mathrm{e}^{4 \kappa_{(5)} \phi(0)}}
$$

It is clear now, that the magnitude of $\epsilon_{v a c}$ is unimportant, as it can be compensated by a shift of $\phi$ : if for some $\epsilon_{v a c}$ there exists a solution with four-dimensional Poincaré invariance, then such a solution exists for any $\epsilon_{v a c}$ of the same sign.

There indeed exist solutions to the resulting system of equations, which have fourdimensional Poincaré invariance [106, 107]. In particular, for $b=3$, these are the only solutions with maximum four-dimensional symmetry [106]: solutions with four-dimensional de Sitter or anti-de Sitter symmetry are absent. For $b=3$, the explicit form of metric is

$$
d s^{2}=\hat{a}^{2}(z) \eta_{\mu \nu} d x^{\mu} d x^{\nu}-d z^{2}
$$


where the warp factor is

$$
\hat{a}(z)=\left(1-\frac{2}{3 \kappa_{(5)}^{2 / 3}} \mathrm{e}^{2 \kappa_{(5)} \tilde{\phi}_{0}} \cdot|z|\right)^{1 / 4}
$$

and $\tilde{\phi}_{0}$ is an arbitrary parameter of solution related to the value of $\phi(z=0)$ as follows,

$$
\tilde{\phi}_{0}=\phi(0)+\frac{1}{2 \kappa_{(5)}} \ln \left(\epsilon_{v a c} \kappa_{(5)}^{8 / 3}\right)
$$

Induced metric on the brane is flat irrespectively of the value of $\epsilon_{v a c}$.

One problem with this mechanism is that metric (60) has a naked singularity at finite proper distance to the brane,

$$
\left|z_{\text {sing }}\right|=\frac{3}{2} \kappa_{(5)}^{2 / 3} \mathrm{e}^{-2 \kappa_{(5)}}
$$

It has been argued that a possible resolution of this singularity reintroduces the cosmological constant problem [111, 112, 113. As an example, if one introduces another brane placed at $|z|<\left|z_{\text {sing }}\right|$ (and imposes the orbifold symmetry), the singularity will be absent between the branes, i.e., in the entire orbifold. However, the tension of the second brane has to be fine-tuned for the metric (60) to remain the solution of the complete set of the Einstein equations. This fine-tuning is no better than the usual fine-tuning of the cosmological constant in four-dimesnional theories.

Another potential difficulty, common to many attempts to solve the cosmological constant problem, is that in the presence of matter, the energy density (in our context, energy density on the brane) contains the vacuum contribution and the contribution of real matter. It is hard to find any distinction between the two; in other words, the same mechanism that compensates for the cosmological constant will tend to modify the gravitational interactions of ordinary matter. This causes doubts that the gravitational interactions in these models reduce to four-dimensional General Relativity at large distances, and, in particular, that any compensation mechanism will be cosmologically viable (see, however, Ref. [114]).

\subsection{Gravity at ultra-large distances}

In relatively simple models discussed in Sections 5 and 6, gravity on the brane is effectively four-dimensional at large enough distances due to the presence of the graviton zero mode. One may wonder whether in other cases the graviton spectrum may be more complicated, and, in particular, whether four-dimensional gravity may be modified at ultra-large scales.

Original (and failed, see below) proposals [70, 115, 116], exploring this possibility, invoked dynamical negative tension branes (and one extra dimension). One of them [115 was to

consider two positive-tension branes of equal tensions $\sigma$ and a negative-tension brane of tension $(-\sigma)$ in between the two. For simplicity, the positive-tension branes are placed at fixed points of an orbifold; in any case, extra dimension is compact. With negative bulk 
cosmological constant tuned according to eq. (27), the warp factor has a minimum at the position $z_{-}$of the negative-tension brane,

$$
a(z)=\mathrm{e}^{k\left|z-z_{-}\right|}, \quad 0 \leq z \leq z_{c}
$$

where $z=0$ and $z=z_{c}$ are positions of the two positive-tension branes. In the limit $z_{c} \rightarrow \infty$, $z_{-} \rightarrow \infty,\left(z_{c}-z_{-}\right) \rightarrow \infty$, all the three branes are infinitely far apart, and there exist two graviton zero modes concentrating near $z=0$ and $z=z_{c}$, respectively. At finite, but fairly large $z_{-}$and $z_{c}$, namely $z_{c}, z_{-},\left(z_{c}-z_{-}\right) \gg k^{-1}$, the degeneracy between the graviton modes is lifted, as is usually the case in quantum mechanics. One linear combination of the modes remains massless, whereas another linear combination acquires a mass $m_{g}$ which is much smaller than the masses of $\mathrm{KK}$ excitations, $m_{K K}$. Hence, at intermediate distances, $m_{K K}^{-1} \ll r \ll m_{g}^{-1}$, both the lightest and massless gravitons contribute into the four-dimensional Newton's law, while at ultra-large distances, $r \gg m_{g}^{-1}$, only massless graviton does (in fact, the situation is more subtle, as there exists a "ghost" massless scalar mode that also contributes into interactions at ultra-large distances, see below). The fourdimensional gravity gets modified at distance $m_{g}^{-1}$, which may be naturally of order than $100 \mathrm{Mpc}$ or larger.

Another set up [70, 116] contains a brane of positive tension $\sigma$ and another brane of negative tension $(-\sigma / 2)$. The bulk cosmological constant between the branes is again tuned as in eq. (27), but $\Lambda$ is set equal to zero outside the negaive-tension brane. Then the four-dimensionally flat solution has a non-trivial warp factor between the branes,

$$
a(z)=\mathrm{e}^{-k|z|}, \quad 0 \leq z \leq z_{-}
$$

whereas the five-dimensional space-time is flat outside the negative-tension brane,

$$
a(z)=\text { const }, \quad z>z_{-}
$$

This set up has infinite extra dimension.

In the limit $z_{-} \rightarrow \infty$, this set up reduces to RS2, and there exists the graviton zero mode near the positive-tension brane. For finite $z_{-}$, this would-be zero mode becomes a resonance of small but finite width $\Gamma_{g}$ [116, 117, 118], i.e., the four-dimensional graviton becomes metastable against escape into extra dimension. At intermediate distances, $k^{-1} \ll r \ll \Gamma_{g}^{-1}$, gravity on the positive-tension brane is four-dimensional. At ultra-large distances, $r \gg \Gamma_{g}^{-1}$, Newton's gravity law is no longer valid; in fact, attraction between masses changes into repulsion at $r \sim \Gamma_{g}^{-1}[119,120$.

The problem [121] with these two scenarios (as well as with intermediate ones [122]) is the existence of dynamical ("vibrating") negative-tension brane(s). It has been shown explicitly [68], in the context of the second set up, that among the excitations about the classical solution, there exists a four-dimensional scalar field with negative kinetic term, i.e., that energy is not positive-definite and, furthermore, is not bounded from below. At quantum level, spontaneous creation of negative energy quanta is possible, so the whole set up is unstable. 
Another way [118, 123] to see that modification of gravity at ultra-large distances is difficult, is to recall the van Dam-Veltman-Zakharov phenomenon [124, 125]. Namely, effective four-dimensional description of gravity with non-trivial properties at ultra-large distances would involve tensor field(s) with small but non-vanishing mass (or width). However, the propagator of massive tensor field in flat background does not coincide with the massless propagator even for $p \gg m$ : the (relevant part of the) former is

$$
G_{\mu \nu \lambda \rho}^{(m)}(p \gg m)=\frac{1}{p^{2}}\left[\frac{1}{2}\left(\eta_{\mu \lambda} \eta_{\nu \rho}+\eta_{\mu \rho} \eta_{\nu \lambda}\right)-\frac{1}{3} \eta_{\mu \nu} \eta_{\lambda \rho}\right]
$$

whereas the latter has another tensor structure,

$$
G_{\mu \nu \lambda \rho}^{(0)}=\frac{1}{p^{2}}\left[\frac{1}{2}\left(\eta_{\mu \lambda} \eta_{\nu \rho}+\eta_{\mu \rho} \eta_{\nu \lambda}\right)-\frac{1}{2} \eta \mu \nu \eta_{\lambda \rho}\right]
$$

Hence, even at intermediate distances, a theory with massive graviton differs from General Relativity; in particular, the former would lead to wrong (and phenomenologically unacceptable) prediction for bending of light [118, 123. In flat background, the correct tensor structure may be restored by adding a "ghost" scalar field with negative kinetic term, which interacts with the trace of energy-momentum tensor with appropriate strength $(1 / 6)$. This is precisely what happens in the above scenarios.

A way out is to consider curved four-dimensional backgrounds. As an example, a theory with massive graviton in four-dimensional anti-de Sitter space-time has correct tensor structure of the propagator, provided the graviton mass is smaller than the inverse adS radius 126, 127 (see, however, Ref. 128]). In the context of extra dimensions, curved fourdimensional space-time emerges when the condition (27) is relaxed. Indeed, it has been found in Refs. [129, 130] that even in models with positive-tension branes only, relaxing the condition (27) leads to four-dimensional gravity which differs from General Relativity at ultra-large scales ("behind the horizon"). It remains to be understood whether this property has observable and/or cosmologically significant consequences.

\subsection{Approximate Lorentz invariance}

Extra dimensions provide, among other things, a framework for studying whether small violation of Lorentz invariance is possible, and what phenomenological consequences it may have. It has been understood long ago [131] that the geometry of the multi-dimensional space-time may not have four-dimensional Lorentz invariance, and yet the four-dimensional geometry induced on "our" brane may be invariant under Lorentz symmetry.

As an example, in five dimensions, the warp factors of time and three-dimensional space may be different, so the metric may have the form

$$
d s^{2}=a^{2}(z) d t^{2}-b^{2}(z) d \mathbf{x}^{2}-d z^{2}
$$

Classical solutions with $a(z) \neq b(z)$ have been found in Refs. [131, 132, 133, 134, 135]; these involve additional matter in the bulk. The four-dimesnional coordinates may be chosen in 
such a way that at the brane position, $z=0$, the warp factors are

$$
a(0)=b(0)=1
$$

and the four-dimensional metric induced on the brane is Minkowskian. If the wave functions of "our" particles have zero width along the fifth direction, it is this Minkowski metric that governs the dynamics of these particles, so the effective four-dimensional theory is exactly Lorentz-invariant. If, however, the wave functions have finite extent in extra dimension, deviations of $a(z)$ and $b(z)$ from 1 are felt by particles on the brane, and four-dimensional Lorentz invariance is only approximate. This, in particular, applies to gravitons: if $a(z)$ (not necessarily $b(z)$ ) decreases sufficiently rapidly towards $z \rightarrow \infty$, gravitons are bound to the brane by essentially the same mechanism as in RS2 set up, and the wave function of the graviton zero mode has finite spread in the fifth dimension.

One Lorentz-violating effect that occurs at relatively low spatial momenta is that the dispersion relation gets modified: instead of the usual $\omega^{2}=m^{2}+\mathbf{p}^{2}$ (where $\omega$ and $\mathbf{p}$ are energy and three-momentum of a particle on the brane), the dispersion relation is now

$$
\omega^{2}=m^{2}+c^{2} \mathbf{p}^{2}
$$

where the parameter $c$ depends on the spread of the wave function in the fifth dimension [133, 134]. The form of the dispersion relation (62) is in accord with the general analysis of Ref. [136]. To see how eq. (62) appears, let us again consider a prototype model of a scalar field with action (48), but now in the background metric (61). The equation for the wave function $\phi$ ant energy $\omega$ is now

$$
\frac{b^{3}(z)}{a(z)} \omega^{2} \phi=a(z) b(z) \mathbf{p}^{2} \phi+\mathcal{H}(z) \phi
$$

where

$$
\mathcal{H}=-\partial_{z}\left(a b^{3} \partial_{z}\right)+a b^{3} V(z)
$$

Note that $\mathcal{H}$ is Hermitean. Let us assume that at $\mathbf{p}=0$, equation (63) has a discrete eigenvalue $\omega^{2}=m^{2}$; the corresponding wave function $\phi_{m}(z)$ concentrates near the brane. At small but non-vanishing $\mathbf{p}$, one considers the first term on the right hand side of eq. (63) as perturbation, and obtains the lowest order correction to the energy,

$$
\Delta \omega^{2}=c^{2} \mathbf{p}^{2}
$$

where

$$
c^{2}=\frac{\int d z a b\left|\phi_{m}(z)\right|^{2}}{\int d z a^{-1} b^{3}\left|\phi_{m}(z)\right|^{2}}
$$

For $a(z) \neq b(z)$, the parameter $c$ is non-universal, as it depends on the shape of the wave function $\phi_{m}$. For narrow wave functions, one has

$$
c^{2}=1+2\left[a^{\prime}(0)-b^{\prime}(0)\right] \frac{\int d z z\left|\phi_{m}(z)\right|^{2}}{\int d z\left|\phi_{m}(z)\right|^{2}}
$$


The violation of Lorentz invariance is small provided the correction to the relation $c^{2}=1$ is smally. The graviton wave function is not, however, narrow in this set up, so the violation of Lorentz invariance in gravitational sector may be substantial. Note that the modification of the dispersion law may take place for both massive and massless particles.

Another effect [135] occurs if $a(z)$ and $b(z)$ have different asymptotics as $|z| \rightarrow \infty$. Suppose that $a(z)$ decreases faster than $b(z)$, i.e.,

$$
a(z) \rightarrow 0, \frac{a(z)}{b(z)} \rightarrow 0 \quad \text { as }|z| \rightarrow \infty
$$

Suppose further that $V(z)$ tends to a constant as $|z| \rightarrow \infty$. Then, in complete analogy to subsection 6.2, the continuum spectrum of eigenvalues $\omega^{2}$ of eq. (63) starts from zero (the term proportional to $V(z)$ is irrelevant at large $|z|$ since $a b^{3} V \ll\left(b^{3} / a\right) \omega^{2}$ at $|z| \rightarrow \infty$ for arbitrarily small $\left.\omega^{2}\right)$. For $\mathbf{p}^{2}=0$, there may exist a localized zero mode which would describe a massless particle residing on a brane. Unlike in subsection 6.2, however, the continuum starts from zero also for $\mathbf{p}^{2} \neq 0$ : the term involving $\mathbf{p}^{2}$ in eq. (63) is also negligible as compared to $\left(b^{3} / a\right) \omega^{2}$ at large $|z|$. On the other hand, the zero mode gets lifted: real part of its energy is equal to $\omega=c|\mathbf{p}|$. Hence, this mode becomes quasi-localized for $\mathbf{p}^{2} \neq 0$ (there are no bound states embedded in continuum). This means that even massless particles are metastable in this set up against escape into extra dimension, provided the corresponding fields have bulk modes. The larger the three-momentum $|\mathbf{p}|$, the larger the width $\Gamma(|\mathbf{p}|)$ [135]. This mechanism of metastability of moving particles in theories with broken four-dimensional Lorentz invariance may have interesting phenomenological consequences, especially for physics of ultra-high energy cosmic rays.

\subsection{Creation of a brane Universe}

At the late stage of the evolution of the Universe, corresponding to temperatures well below the maximum temperature $T_{*}$ in ADD scenario or well below $\mathrm{TeV}$ in RS1 and RS2 models, brane cosmology is governed by the same four-dimensional laws as the standard FRW cosmology [137, 138, 139] (in the case of RS1, this assertion holds only if the radion is stabilized, otherwise the late cosmology is very unconventional [140, 141]). An important assumption here is that the bulk is empty, and, in the case of infinite extra dimensions, that the horizon "does not shine" on our brane. Are these assumptions reasonable? Is it conceivable that our world with all its reach structure is merely a brane embedded in empty multi-dimensional space?

This brings us to a problem of the beginning of the brane Universe. The requirement that the bulk should be essentially empty suggests a picture of spontaneous creation of "our" brane in a multi-dimensional space which initially has neither branes nor other excitations [142. A model in which this possibility is realized is a five-dimensional theory of fourform field (four-index anti-symmetric tensor field) $B$ and branes, both charged and neutral

\footnotetext{
${ }^{9}$ Another possibility is that the wave functions of all particles bound to the brane are very similar.
} 
with respect to this field. Just like a charged particle couples to a vector field through its world-line, $S_{i n t}=e \int A_{\mu} d x^{\mu}$, a charged brane couples to the four-form field through its four-dimensional world-volume,

$$
S_{i n t}=e \int B_{A B C D} d \Omega^{A B C D}
$$

The picture is then as follows. Suppose that an initial state of a five-dimensional Universe contains the $B$-field of constant field strength,

$$
H_{A B C D E} \equiv \partial_{[A} B_{B C D E]}=h \sqrt{g^{(5)}} \epsilon_{A B C D E}
$$

If it were not for the charged branes, the field $B$ would act as a five-dimensional cosmological constant, as its field equations would require that

$$
h=\text { const }
$$

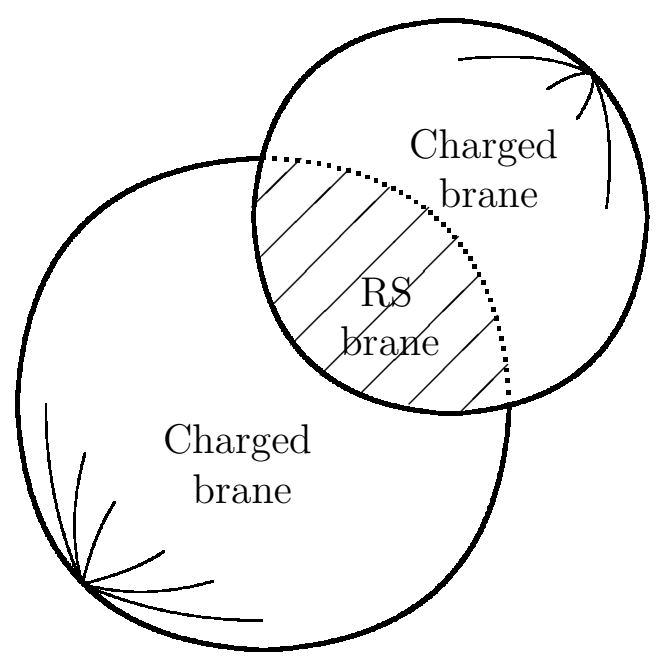

Figure 10: Spontaneously created system of two charged branes with neutral (RS) brane between them.

For non-vanishing $h$, charged branes are created spontaneously [143, much in the same way as $e^{+} e^{-}$-pairs are spontaneously created by background electric field. Furthermore, a neutral brane has a chance to be created in between the charged ones [142]; it is this neutral brane that is interpreted as our Universe. The system of the three branes at the moment of their materialization is shown in Fig. 10. After spontaneous creation, the three-volumes of all the branes increase, the junction between the neutral and charged branes moves away, and most of the neutral brane becomes a homogeneous three-dimensional space. The bulk surrounding it remains empty. 
If $h$ is not large, the probability of brane creation is exponentially small. So, it is natural that other branes, which may be created in the five-dimensional space, are extremely far away from "ours". Also, with sufficiently complicated theory on "our" brane, it is conceivable that its initial state is such that a period of the usual four-dimensional inflation on this brane occurs after it is created. Hence, the model of Ref. [142] is capable of reproducing the conventional cosmological picture, with our Universe being a brane embedded in empty bulk. This model certainly represents an interesting twist in the discussion of the origin of our Universe.

\section{Conclusion}

Theories with large and infinite extra dimensions look rather exotic, at least for the moment. Yet they lead to important insights on what unusual phenomena may occur both at the energy scales accessible to future accelerators, and at low energies, the domain of possible rare effects. They also provide a framework for addressing a number of phenomenological issues, like consistency of apparent non-coneservation of energy and electric charge, naturalness of small violation of Lorentz invariance and possible manifestations of a strongly coupled conformal sector which interacts weakly with the Standard Model fields. Furthermore, new ideas emerge in approaching fundamental problems, such as the cosmological constant problem or the beginning of our Universe.

All this makes the subject interesting and lively. The question is whether Nature follows any of the routes being explored in this context.

The author is indebted to L. B. Okun for his suggestion to write this mini-review. He thanks numerous colleagues at INR and elsewhere for illuminating discussions. This work

has been supported in part by the Council for Presidential Grants and State Support of Leading Scientific Schools, grant 00-15-96626, RFBR grant 99-01-18410 and Swiss Science Foundation Grant 7SUPJ062239.

\section{References}

[1] I. P. Volobuev and Y. A. Kubyshin, Theor. Math. Phys. 68 (1986) 788; Theor. Math. Phys. 68 (1986) 885; JETP Lett. 45 (1987) 581.

[2] I. Antoniadis, Phys. Lett. B 246 (1990) 377.

[3] J. D. Lykken, Phys. Rev. D 54 (1996) 3693 hep-th/9603133.

[4] J. Polchinski, "TASI lectures on D-branes," hep-th/9611050.

[5] P. Horava and E. Witten, Nucl. Phys. B 460 (1996) 506 hep-th/9510209.

[6] A. Lukas, B. A. Ovrut, K. S. Stelle and D. Waldram, Phys. Rev. D 59 (1999) 086001 hep-th/9803235. 
[7] M. Visser, "An Exotic Class Of Kaluza-Klein Models," hep-th/9910093.

[8] V. A. Rubakov and M. E. Shaposhnikov, Phys. Lett. B 125 (1983) 136.

[9] K. Akama, Lect. Notes Phys. 176 (1982) 267 hep-th/0001113.

[10] R. Jackiw and C. Rebbi, Phys. Rev. D 13 (1976) 3398.

[11] R. Jackiw and P. Rossi, Nucl. Phys. B 190 (1981) 681.

[12] G. 't Hooft, Phys. Rev. D 14 (1976) 3432.

[13] M. V. Libanov and S. V. Troitsky, Nucl. Phys. B 599 (2001) 319 hep-ph/0011095.

[14] J. M. Frere, M. V. Libanov and S. V. Troitsky, "Three generations on a local vortex in extra dimensions," hep-ph/0012306.

[15] G. Dvali and M. Shifman, Phys. Lett. B 396 (1997) 64 [hep-th/9612128].

[16] G. Dvali, G. Gabadadze and M. Porrati, Phys. Lett. B 485 (2000) 208 hep-th/0005016.

[17] G. Dvali and G. Gabadadze, Phys. Rev. D 63 (2001) 065007 hep-th/0008054.

[18] G. Dvali, G. Gabadadze and M. Shifman, Phys. Lett. B 497 (2001) 271 hepth/0010071.

[19] S. L. Dubovsky and V. A. Rubakov, to appear

[20] F. Quevedo and C. A. Trugenberger, Nucl. Phys. B 501 (1997) 143 hep-th/9604196;

. M. Polyakov, Nucl. Phys. B 486 (1997) 23 [hep-th/9607049.

[21] N. Arkani-Hamed, S. Dimopoulos and G. Dvali, Phys. Lett. B 429 (1998) 263 hepph/9803315.

[22] I. Antoniadis, N. Arkani-Hamed, S. Dimopoulos and G. Dvali, Phys. Lett. B 436 (1998) 257 [hep-ph/9804398].

[23] C. D. Hoyle, U. Schmidt, B. R. Heckel, E. G. Adelberger, J. H. Gundlach, D. J. Kapner and H. E. Swanson, Phys. Rev. Lett. 86 (2001) 1418 [hep-ph/0011014].

[24] V. P. Mitrofanov and O. I. Ponomareva, JETP 67 (1988) 1963.

[25] Y. Su, B. R. Heckel, E. G. Adelberger, J. H. Gundlach, M. Harris, G. L. Smith and H. E. Swanson, Phys. Rev. D 50 (1994) 3614.

[26] J. C. Long, H. W. Chan and J. C. Price, Nucl. Phys. B 539 (1999) 23 hep-ph/9805217.

[27] J. C. Long, A. B. Churnside and J. C. Price, hep-ph/0009062. 
[28] V. B. Braginsky, private communication.

[29] G. F. Giudice, R. Rattazzi and J. D. Wells, Nucl. Phys. B 544 (1999) 3 hepph/9811291.

[30] G. F. Giudice, R. Rattazzi and J. D. Wells, Nucl. Phys. B 595 (2001) 250 hepph/0002178.

[31] S. Nussinov and R. Shrock, Phys. Rev. D 59 (1999) 105002 hep-ph/9811323.

[32] J. L. Hewett, Phys. Rev. Lett. 82, 4765 (1999) hep-ph/9811356.

[33] T. Han, J. D. Lykken and R. Zhang, Phys. Rev. D 59 (1999) 105006 hep-ph/9811350.

[34] N. Arkani-Hamed, S. Dimopoulos and G. Dvali, Phys. Rev. D 59 (1999) 086004 [hepph/9807344.

[35] N. Kaloper, J. March-Russell, G. D. Starkman and M. Trodden, Phys. Rev. Lett. 85 (2000) 928 [hep-ph/0002001.

[36] Z. Kakushadze and S. H. Tye, Nucl. Phys. B 548 (1999) 180 hep-th/9809147.

[37] G. Shiu, R. Shrock and S. H. Tye, Phys. Lett. B 458 (1999) 274 hep-ph/9904262.

[38] S. Cullen, M. Perelstein and M. E. Peskin, Phys. Rev. D 62 (2000) 055012 hepph/0001166.

[39] G. F. Giudice, E. W. Kolb and A. Riotto, "Largest temperature of the radiation era and its cosmological implications," hep-ph/0005123.

[40] S. Cullen and M. Perelstein, Phys. Rev. Lett. 83 (1999) 268 hep-ph/9903422.

[41] V. Barger, T. Han, C. Kao and R. J. Zhang, Phys. Lett. B 461 (1999) 34 [hepph/9905474

[42] C. Hanhart, D. R. Phillips, S. Reddy and M. J. Savage, Nucl. Phys. B 595 (2001) 335 nucl-th/0007016.

[43] S. Hannestad and G. Raffelt, "New Supernova Limit on Large Extra Dimensions," hep-ph/0103201.

[44] N. Arkani-Hamed, S. Dimopoulos, G. Dvali and J. March-Russell, "Neutrino masses from large extra dimensions," hep-ph/9811448.

[45] K. R. Dienes, E. Dudas and T. Gherghetta, Nucl. Phys. B 557 (1999) 25 hepph/9811428.

[46] G. Dvali and A. Y. Smirnov, Nucl. Phys. B 563 (1999) 63 hep-ph/9904211. 
[47] R. N. Mohapatra, S. Nandi and A. Perez-Lorenzana, Phys. Lett. B 466 (1999) 115 hep-ph/9907520;

R. N. Mohapatra and A. Perez-Lorenzana, Nucl. Phys. B 576 (2000) 466 hepph/9910474.

[48] R. Barbieri, P. Creminelli and A. Strumia, Nucl. Phys. B 585 (2000) 28 hepph/0002199.

[49] A. Lukas, P. Ramond, A. Romanino and G. G. Ross, Phys. Lett. B 495 (2000) 136 hep-ph/0008049.

[50] N. Cosme, J. M. Frere, Y. Gouverneur, F. S. Ling, D. Monderen and V. Van Elewyck, "Neutrino suppression and extra dimensions: A minimal model," hep-ph/0010192.

[51] A. Perez-Lorenzana, "Theories in more than four dimensions," hep-ph/0008333.

[52] K. R. Dienes, E. Dudas and T. Gherghetta, Phys. Lett. B 436 (1998) 55 hepph/9803466; Nucl. Phys. B 537 (1999) 47 hep-ph/9806292.

[53] C. P. Bachas, JHEP 9811 (1998) 023 hep-ph/9807415].

[54] I. Antoniadis and C. Bachas, Phys. Lett. B 450 (1999) 83 hep-th/9812093.

[55] N. Arkani-Hamed, S. Dimopoulos and J. March-Russell, hep-th/9908146.

[56] L. M. Krauss and F. Wilczek, Phys. Rev. Lett. 62 (1989) 1221.

[57] M. G. Alford, J. March-Russell and F. Wilczek, Nucl. Phys. B 337 (1990) 695.

[58] J. Preskill and L. M. Krauss, Nucl. Phys. B 341 (1990) 50.

[59] M. G. Alford, S. Coleman and J. March-Russell, Nucl. Phys. B 351 (1991) 735.

[60] M. Kamionkowski and J. March-Russell, Phys. Lett. B 282 (1992) 137 [hepth/9202003.

[61] W. Israel, Nuovo Cim. B 44S10 (1966) 1.

[62] V. A. Berezin, V. A. Kuzmin and I. I. Tkachev, Phys. Rev. D 36 (1987) 2919.

[63] D. Z. Freedman, S. S. Gubser, K. Pilch and N. P. Warner, JHEP0007 (2000) 038 hep-th/9906194.

[64] M. Gogberashvili, Europhys. Lett. 49 (2000) 396 hep-ph/9812365.

[65] M. Gogberashvili, Mod. Phys. Lett. A 14 (1999) 2025 hep-ph/9904383.

[66] L. Randall and R. Sundrum, Phys. Rev. Lett. 83 (1999) 3370 hep-ph/9905221. 
[67] L. Randall and R. Sundrum, Phys. Rev. Lett. 83 (1999) 4690 hep-th/9906064.

[68] L. Pilo, R. Rattazzi and A. Zaffaroni, JHEP0007 (2000) 056 [hep-th/0004028].

[69] C. Csaki, M. Graesser, L. Randall and J. Terning, Phys. Rev. D 62 (2000) 045015 hep-ph/9911406.

[70] C. Charmousis, R. Gregory and V. A. Rubakov, Phys. Rev. D 62 (2000) 067505 [hepth/9912160.

[71] W. D. Goldberger and M. B. Wise, Phys. Rev. Lett. 83 (1999) 4922 [hep-ph/9907447].

[72] M. A. Luty and R. Sundrum, Phys. Rev. D 62 (2000) 035008 hep-th/9910202.

[73] J. Garriga and T. Tanaka, Phys. Rev. Lett. 84 (2000) 2778 hep-th/9911055.

[74] S. B. Giddings, E. Katz and L. Randall, JHEP 0003 (2000) 023 [hep-th/0002091].

[75] H. Davoudiasl, J. L. Hewett and T. G. Rizzo, Phys. Rev. Lett. 84 (2000) 2080 hepph/9909255.

[76] N. Arkani-Hamed, M. Porrati and L. Randall, "Holography and phenomenology," hepth/0012148.

[77] T. Gherghetta and A. Pomarol, "A warped supersymmetric standard model," hepph/0012378.

[78] E. T. Akhmedov, "Introduction to the AdS/CFT correspondence," hep-th/9911095, to appear in Uspekhi Fiz. Nauk.

[79] W. Muck, K. S. Viswanathan and I. V. Volovich, Phys. Rev. D 62 (2000) 105019 hep-th/0002132].

[80] R. Gregory, V. A. Rubakov and S. M. Sibiryakov, Class. Quant. Grav. 17 (2000) 4437 hep-th/0003109.

[81] J. Lykken and L. Randall, JHEP0006 (2000) 014 hep-th/9908076.

[82] A. Hebecker and J. March-Russell, "Randall-Sundrum II cosmology, AdS/CFT, and the bulk black hole," hep-ph/0103214.

[83] S. L. Dubovsky, V. A. Rubakov and P. G. Tinyakov, Phys. Rev. D 62 (2000) 105011 hep-th/0006046.

[84] B. Bajc and G. Gabadadze, Phys. Lett. B 474 (2000) 282 [hep-th/9912232.

[85] J. Maldacena, Adv. Theor. Math. Phys. 2 (1998) 231 hep-th/9711200]. 
[86] S. S. Gubser, I. R. Klebanov and A. M. Polyakov, Phys. Lett. B 428 (1998) 105 [hepth/9802109.

[87] E. Witten, Adv. Theor. Math. Phys. 2 (1998) 505 hep-th/9803131.

[88] H. Verlinde, Talk at ITP Santa Barbara conference "New Dimensions in Field Theory and String Theory", http://www.itp.ucsb.edu/online/susy_c99/verlinde;

E. Witten, ibid., http://www.itp.ucsb.edu/online/susy_c99/discussion .

[89] S. S. Gubser, "AdS/CFT and gravity," hep-th/9912001.

[90] S. B. Giddings and E. Katz, hep-th/0009176.

[91] S. Coleman and L. Smarr, Commun. Math. Phys. 56 (1977) 1.

[92] S. W. Hawking, T. Hertog and H. S. Reall, Phys. Rev. D 62 (2000) 043501 [hepth/0003052.

[93] S. Nojiri and S. D. Odintsov, Phys. Lett. B 484 (2000) 119 [hep-th/0004097.

[94] L. Anchordoqui, C. Nunez and K. Olsen, JHEP 0010 (2000) 050 hep-th/0007064.

[95] N. Arkani-Hamed, S. Dimopoulos, G. Dvali and N. Kaloper, Phys. Rev. Lett. 84 (2000) 586 [hep-th/9907209].

[96] T. Gherghetta and M. Shaposhnikov, Phys. Rev. Lett. 85 (2000) 240 hep-th/0004014].

[97] A. G. Cohen and D. B. Kaplan, Phys. Lett. B 470 (1999) 52 hep-th/9910132.

[98] R. Gregory, Phys. Rev. Lett. 84 (2000) 2564 hep-th/9911015.

[99] T. Gherghetta, E. Roessl and M. Shaposhnikov, Phys. Lett. B 491 (2000) 353 [hepth/0006251.

[100] S. Randjbar-Daemi and M. Shaposhnikov, Phys. Lett. B 491 (2000) 329 [hepth/0008087.

[101] S. L. Dubovsky, V. A. Rubakov and P. G. Tinyakov, JHEP0008 (2000) 041 [hepph/0007179.

[102] I. Oda, Phys. Lett. B 496 (2000) 113 hep-th/0006203].

[103] E. Ponton and E. Poppitz, JHEP0102 (2001) 042 hep-th/0012033.

[104] V. A. Rubakov and M. E. Shaposhnikov, Phys. Lett. B 125 (1983) 139.

[105] S. Randjbar-Daemi and C. Wetterich, Phys. Lett. B 166 (1986) 65. 
[106] N. Arkani-Hamed, S. Dimopoulos, N. Kaloper and R. Sundrum, Phys. Lett. B 480 (2000) 193 hep-th/0001197.

[107] S. Kachru, M. Schulz and E. Silverstein, Phys. Rev. D 62 (2000) 085003 [hepth/0002121].

[108] A. D. Dolgov, In *Cambridge 1982, Proceedings, The Very Early Universe*, 449-458.

[109] R. D. Peccei, J. Sola and C. Wetterich, Phys. Lett. B 195 (1987) 183.

[110] S. M. Barr and D. Hochberg, Phys. Lett. B 211 (1988) 49.

[111] S. P. de Alwis, Nucl. Phys. B 597 (2001) 263 hep-th/0002174;

S. P. de Alwis, A. T. Flournoy and N. Irges, JHEP0101 (2001) 027 hep-th/0004125.

[112] S. Forste, Z. Lalak, S. Lavignac and H. P. Nilles, Phys. Lett. B 481 (2000) 360 [hepth/0002164.

[113] C. Csaki, J. Erlich, C. Grojean and T. Hollowood, Nucl. Phys. B 584 (2000) 359 hep-th/0004133.

[114] V. A. Rubakov, Phys. Rev. D 61 (2000) 061501 hep-ph/9911305.

[115] I. I. Kogan, S. Mouslopoulos, A. Papazoglou, G. G. Ross and J. Santiago, Nucl. Phys. B 584 (2000) 313 hep-ph/9912552.

[116] R. Gregory, V. A. Rubakov and S. M. Sibiryakov, Phys. Rev. Lett. 84 (2000) 5928 hep-th/0002072].

[117] C. Csaki, J. Erlich and T. J. Hollowood, Phys. Rev. Lett. 84 (2000) 5932 [hepth/0002161].

[118] G. Dvali, G. Gabadadze and M. Porrati, Phys. Lett. B 484 (2000) 112 [hepth/0002190].

[119] R. Gregory, V. A. Rubakov and S. M. Sibiryakov, Phys. Lett. B 489 (2000) 203 hep-th/0003045.

[120] C. Csaki, J. Erlich, T. J. Hollowood and J. Terning, Phys. Rev. D 63 (2001) 065019 hep-th/0003076.

[121] E. Witten, "The cosmological constant from the viewpoint of string theory," hepph/0002297.

[122] I. I. Kogan and G. G. Ross, Phys. Lett. B 485 (2000) 255 hep-th/0003074.

[123] G. Dvali, G. Gabadadze and M. Porrati, Phys. Lett. B 484 (2000) 129 [hepth/0003054. 
[124] H. van Dam and M. Veltman, Nucl. Phys. B 22 (1970) 397.

[125] V. I. Zakharov, JETP Lett. 12, 312 (1970).

[126] I. I. Kogan, S. Mouslopoulos and A. Papazoglou, Phys. Lett. B 503 (2001) 173 [hepth/0011138].

[127] M. Porrati, Phys. Lett. B 498 (2001) 92 [hep-th/0011152].

[128] F. A. Dilkes, M. J. Duff, J. T. Liu and H. Sati, "Quantum M -i, 0 discontinuity for massive gravity with a Lambda term," hep-th/0102093.

[129] I. I. Kogan, S. Mouslopoulos and A. Papazoglou, Phys. Lett. B 501 (2001) 140 [hepth/0011141].

[130] A. Karch and L. Randall, "Locally localized gravity," hep-th/0011156.

[131] M. Visser, Phys. Lett. B 159 (1985) 22.

[132] P. Bowcock, C. Charmousis and R. Gregory, Class. Quant. Grav. 17 (2000) 4745 hep-th/0007177.

[133] D. J. Chung, E. W. Kolb and A. Riotto, "Extra dimensions present a new flatness problem," hep-ph/0008126.

[134] C. Csaki, J. Erlich and C. Grojean, "Gravitational Lorentz violations and adjustment of the cosmological constant in asymmetrically warped spacetimes," hep-th/0012143.

[135] S. L. Dubovsky, "Tunneling into extra dimension and high-energy violation of Lorentz invariance," hep-th/0103205.

[136] S. Coleman and S. L. Glashow, Phys. Rev. D 59 (1999) 116008 hep-ph/9812418.

[137] C. Csaki, M. Graesser, C. Kolda and J. Terning, Phys. Lett. B 462 (1999) 34 [hepph/9906513.

[138] J. M. Cline, C. Grojean and G. Servant, Phys. Rev. Lett. 83 (1999) 4245 [hepph/9906523.

[139] C. Csaki, M. Graesser, L. Randall and J. Terning, Phys. Rev. D 62 (2000) 045015 hep-ph/9911406.

[140] P. Binetruy, C. Deffayet and D. Langlois, Nucl. Phys. B 565 (2000) 269 hepth/9905012].

[141] D. J. Chung and K. Freese, Phys. Rev. D 61 (2000) 023511 hep-ph/9906542. 
[142] A. Gorsky and K. Selivanov, Phys. Lett. B 485 (2000) 271 hep-th/0005066; "Brane tunneling and the brane world scenario," hep-th/0009207.

[143] J. D. Brown and C. Teitelboim, Nucl. Phys. B 297 (1988) 787. 